\title{
Achieving high-speed retraction in a stretchable
} University, MS State, MS 39762, USA.

7 KEYWORDS: Resilient gels, Bioinspired, Highly stretchable gels, Hydrophobic interactions,

8 Retraction velocity 
1 ABSTRACT: Hydrogels mimicking elastomeric biopolymers such as resilin, responsible for

2 power amplified activities in biological species necessary for locomotion, feeding, and defense,

3 can have applications in soft-robotics and prosthetics. Here, we report a bioinspired hydrogel

4 synthesized through a free radical polymerization reaction. By maintaining a balance between the

5 hydrophilic and hydrophobic components, we obtain gels with elastic modulus as high as $100 \mathrm{kPa}$,

6 stretchability up to $800 \%$, and resilience up to $98 \%$. Such properties enable these gels to catapult

7 projectiles. Further, these gels achieve a retraction velocity of $16 \mathrm{~m} \mathrm{~s}^{-1}$ with an acceleration of

$84 \times 10^{3} \mathrm{~m} \mathrm{~s}^{-2}$ when released from a stretched state, and these values are comparable to those

9 observed in many biological species during the power amplification process. By utilizing and

10 tuning the simple synthetic strategy used here, these gels can be used in soft robotics, prosthetics,

11 and engineered devices where power amplification is desired. 


\section{$1 \quad$ Introduction}

2 Elastomeric biopolymers such as resilin play a vital role in dictating the power amplification

3 process. ${ }^{1-4}$ Biological species perform some remarkable activities necessary for locomotion,

4 feeding, and defense. ${ }^{5}$ Many of these activities include a power amplification mechanism, in which

5 a particular biological system rapidly releases stored-energy by achieving a very high velocity over

6 a short period of time, resulting in high power output. Such power amplification allows insects

7 such as locust to jump and Mantis shrimp to kill prey by its appendage strike. ${ }^{5,6}$ These elastic

8 biopolymers display high and reversible stretchability with low energy dissipation -typically

9 characterized as high resilience- and rapidly retract to their original dimensions after the removal

10 of mechanical load. ${ }^{1,7}$ Specifically, resilin displays up to $300 \%$ stretchability and high resilience,

11 as $>90 \%$ of the applied energy stored during deformation is recovered. ${ }^{1,3,4}$ Such remarkable

12 properties of resilin have been attributed to a balanced combination of entropic and enthalpic

13 elasticity originated from the presence of hydrophilic (exon I) and hydrophobic (exon III) segments

14 in resilin. ${ }^{1-4}$

15 A reasonably good understanding regarding the origin of elasticity in elastomeric biomaterials

16 allows us to engineer materials mimicking some characteristics of those biomaterials. There are

17 many potential applications, viz. in artificial muscles, prosthetic devices for assisting people with

18 restricted mobility, energy harvesting, and enabling soft-robots for power amplification. ${ }^{8}$ Since

19 hydrogels are the natural choice of mimicking water containing biomaterials, there are numerous

20 efforts in developing highly stretchable and resilient hydrogels. ${ }^{9,10}$ However, some of these

21 hydrogels are based on recombinant proteins, therefore, obtaining a significant amount of such

22 materials for practical applications is challenging. ${ }^{1,11}$ There also have been efforts to synthesize

23 polymeric hydrogels with high stretchability and resilience, but many of the synthesis schemes 
1 require complex reaction routes that limit their large-scale applicability. ${ }^{90,12-14}$ Furthermore,

2 although many of these systems display high stretchability, a significant energy dissipation during

3 cyclic loading is observed. ${ }^{9,10,12-14}$ None of these hydrogels have been tested for the retraction

4 behavior from a stretched state, however, the dissipation of energy observed in these gels is 5 expected to cause a lower retraction velocity, which will defeat the very purpose of their 6 applications for power amplification.

7 Here, we report a highly stretchable and resilient hydrogel that displays a very high retraction

8 velocity gained over a short period of time, i.e. with high acceleration, after released from a

9 stretched state. The hydrogels are synthesized through a simple reaction scheme involving free-

10 radical copolymerization of acrylic acid (AAc), methacrylamide (MAM), and poly(propylene

11 glycol) diacrylate (PPGDA) using potassium persulfate (KPS) as an initiator. To the best of our

12 knowledge, a hydrogel capable of achieving such high retraction velocity and acceleration has not

13 been reported in the literature. Because of these attributes, these hydrogels have been demonstrated 14 to catapult projectiles over a long-distance.

\section{Experimental Section}

17 Materials. To synthesize the gels in this study, we have used poly(propylene glycol) diacrylate 18 (PPGDA) with a molecular weight of $800 \mathrm{~g} \mathrm{~mol}^{-1}$, sodium dodecyl sulfate (SDS, 98.5\%), acrylic 19 acid (AAc, 99\%), methacrylamide (MAM, 98\%), and potassium persulfate (KPS, 99\%). All these

20 chemicals were obtained from Sigma Aldrich and used as received. Sodium chloride $(\mathrm{NaCl}$, $2199.9 \%$ ) was purchased from Fisher Scientific and was used as received. For all experiments, 22 deionized water of resistivity $18.2 \mathrm{~m} \Omega$ was used.

23 Hydrogel Synthesis. The gel samples were synthesized with different concentrations of 24 monomers (AAc and MAM). The nomenclature used for the gels (17\%-, 27\%-, 37\%-Gels) 
1 indicates the total concentration of the monomers $\left(\mathrm{g} \mathrm{mL}^{-1}\right)$ used to synthesize the gels. First,

2 aqueous $0.8 \mathrm{M} \mathrm{NaCl}$ solution was prepared. $83 \mathrm{~mL}$ of saline solution was mixed with $7 \%\left(\mathrm{~g} \mathrm{ml}^{-1}\right)$

3 of SDS, considering $100 \mathrm{~mL}$ as the volume basis. The solution was stirred using a magnetic stirrer

4 at $220 \mathrm{rpm}$ for approximately $30 \mathrm{~min}$ in a warm water bath maintained at $55{ }^{\circ} \mathrm{C}$ until a clear

5 solution was observed. The desired amount of monomer mixture was prepared separately by

6 adding MAM into AAc, maintaining a weight ratio of MAM to AAc as 1:4, and considering 100

$7 \mathrm{~mL}$ as a basis for the calculation. The monomer mixture was mixed for approximately $15 \mathrm{~min}$ at

$8220 \mathrm{rpm}$ using a magnetic stirrer at room temperature $\left(22^{\circ} \mathrm{C}\right)$. The clear solution of SDS in saline

9 water was taken out from the warm water bath and mixed with $1 \%\left(\mathrm{~g} \mathrm{~g}^{-1}\right)$ of PPGDA using the

10 monomer mixture weight as a reference. This solution was mixed for 5 min using a magnetic stirrer

11 at $220 \mathrm{rpm}$. After mixing, the monomer mixture was added dropwise to this solution and stirred

12 for an additional 5 minutes to obtain a mixture of reactants. $0.2 \%\left(\mathrm{~g} \mathrm{~g}^{-1}\right)$ of KPS was prepared,

13 considering the monomer mixture as a basis, added into $17 \mathrm{~mL}$ of saline solution, and stirred for 5

$14 \mathrm{~min}$. The KPS solution was added dropwise to the reactant mixture while stirring it at $220 \mathrm{rpm}$.

15 The final transparent solution was poured in an airtight glass container and placed in a water bath

16 at $75{ }^{\circ} \mathrm{C}$ for $2 \mathrm{~h}$ to obtain a translucent gel. The containers used to cast the gels were a $2 \mathrm{~L}$ bottle,

$175 \mathrm{~mL}$ vials, and 5-mm-diameter glass tubes. Once the gels formed, the heat source was turned off,

18 and the water bath was allowed to cool down for the next $24 \mathrm{~h}$ before samples were used for further

19 testing.

20 Swelling Experiments. For the swelling tests, small cubic samples $\left(10 \times 10 \times 10 \mathrm{~mm}^{3}\right)$ were

21 weighed, dried in a vacuum oven $(\approx 226$ torr $)$ for $72 \mathrm{~h}$ at room temperature, and weighed again to

22 determine the water loss. The water content in the as-prepared gels was estimated as the change in

23 the gel weight after drying with respect to the initial sample weight. Next, the samples were 
1 immersed in $50 \mathrm{~mL}$ of DI water for $24 \mathrm{~h}$ to swell. The swelling ratio $\left(\mathrm{g} \mathrm{g}^{-1}\right)$ was obtained as a ratio

2 of sample weight after swelling to the dry weight.

3 Tensile testing. All mechanical tests were performed at room temperature $\left(22^{\circ} \mathrm{C}\right)$. A custom-

4 built setup was used for conducting the tensile experiments, and the details regarding the 5 experimental setup and data analysis protocol can be found in our previous publications. ${ }^{15,16}$ In

6 these experiments, a dogbone-shaped gel sample was prepared by cutting from the gel-sheet using 7 a 3D-printed punch. The dogbone sample had a gauge length of $4.2 \mathrm{~mm}$, breadth of $4.2 \mathrm{~mm}$, and 8 an approximate thickness of $9.5 \mathrm{~mm}$. The dogbone sample was held by the four supporting pins

9 fixed to the top and bottom support blocks. A clamp was attached at the top and bottom blocks to 10 avoid the sample slippage. The sample was stretched by moving the top supporting block using a 11 moving stage (M414.3PD, Physik Instrumente). The sample was stretched with the stage velocity

12 of $1 \mathrm{~mm} \mathrm{~s}^{-1}$ (strain-rate $(\dot{\varepsilon})$ of $0.048 \mathrm{~s}^{-1}$ ) until the failure of gel occurred. The setup was controlled 13 by a custom-built program in NI LabVIEW framework. During the sample stretching, images were 14 taken by a monochrome camera (Grasshopper3, Point Grey Research Inc.) at $\approx 16$ fps. Every 15 sample was marked with three lines $(1,2,3)$ at the gauge region to calculate strain $(\varepsilon)$ and $\dot{\varepsilon}$ by 16 using a custom-built image-processing program in MATLAB. Here, $\varepsilon$ represents the average of 17 the change in distance between lines 1-2, 2-3, and 1-3, normalized by their initial distances. The 18 corresponding stretch ratio, $\lambda$, can be estimated as $\varepsilon+1$. The force value corresponding to an 19 applied stretch is measured by the deflection of a cantilever with a known stiffness. Nominal stress $20(\sigma)$ was estimated by dividing the measured force with the initial cross-sectional area of the gauge 21 region of the sample.

22 Cyclic loading and determining resilience. Cyclic loading experiments were also performed 23 using the custom-built setup described above. In these experiments, samples were stretched with 
1 the same strain-rate $(\dot{\varepsilon})$ of $0.048 \mathrm{~s}^{-1}$ until those experienced the prescribed nominal stress $(\sigma)$.

2 After the prescribed stress was attained, the samples were unloaded with the same strain-rate until

3 the stress became zero. The applied strain and strain-rates were determined from the images as

4 described above in the tensile testing section.

5 Retraction experiments. For the retraction experiments, a gel string sample with a diameter of $64 \mathrm{~mm}$ and a length of $45 \mathrm{~mm}$ was clamped at both ends on the tensile testing setup. The sample

7 was then stretched, and stretch ratios of $\lambda=3,4,5$, and 6 were considered. The sample was then

8 marked with seven equidistant lines that were tracked during the retraction process, as described

9 below. The string was cut with scissors adjacent to the bottom clamp and was allowed to retract.

10 The retraction process was recorded by using a high-speed camera (Miro M310, Phantom) at

$11 \approx 5000 \mathrm{fps}$. The images were analyzed using a custom-developed image-processing program in

12 MATLAB. The data was used from the time at which the string was snapped. While retracting,

13 the string slacks as soon as it crosses its initial length. The data after slack-time was not used for

14 analysis.

15 To calculate the velocity of each marked line, a fifth-order of Fourier series was used to fit the 16 position (f) vs. time $(t)$ data, which can be mathematically represented as, $17 f(t)=a_{0}+\sum_{i=1}^{5} a_{i} \cos (\omega t)+\sum_{i=1}^{5} b_{i} \sin (\omega t)$, where $a_{0}, a_{i} \mathrm{~s}, b_{i}$ s, and $\omega$ are the fitting parameters. The 18 resultant function was then differentiated to obtain the velocity. The differentiation of the above

19 equation can be mathematically expressed as, $\frac{d f(t)}{d t}=\omega\left(-\sum_{i=1}^{5} a_{i} \sin (\omega t)+\sum_{i=1}^{5} b_{i} \cos (\omega t)\right)$. The

20 expression was further differentiated to estimate the acceleration, which can be mathematically

21 represented as, $\frac{d^{2} f(t)}{d t^{2}}=-(\omega)^{2}\left(\sum_{i=1}^{5} a_{i} \cos (\omega t)+\sum_{i=1}^{5} b_{i} \sin (\omega t)\right)$. 


\section{Results and discussion}

2 Hydrogel synthesis. As displayed in Figure 1A, upon chemical reaction, AAc and MAM form 3 hydrophilic poly(acrylic acid) (PAAc) and poly(methacrylamide) (PMAM) chains. The

4 hydrophobic PPGDA chains connect the PAAc and PMAM chains forming crosslinks. Figure 1B

5 represents a schematic of the proposed network structure of the gel. Note that PPGDA chains can

6 also polymerize as a result of the free radical reaction, leading to a significant distribution in their

7 molecular weight. Because of hydrophobicity, the poly(propylene glycol) (PPG) blocks collapse,

8 and multiple of these blocks can associate forming hydrophobic aggregates. The surfactant SDS

9 in the $\mathrm{NaCl}$ reaction media promotes the formation of these hydrophobic aggregates. ${ }^{17}$ The gels

10 are opaque, indicating a phase-separated structure.

11 Previously, gels were synthesized by using free-radical polymerization of acrylic acid (AAc), 12 methacrylamide (MAM), and copolymerization of AAc and MAM, and in many cases N,N'13 methylenebisacrylamide was used as a crosslinker. ${ }^{18-21}$ These gels have displayed high water 14 swelling capabilities. In a limited number of studies, hydrophobic chains have also been 15 incorporated. ${ }^{22,23}$ High stretchability has also been reported in a few studies, but that can be 16 associated with hysteresis. ${ }^{19,22}$ The PPG or poly(propylene oxide) present in the PPGDA becomes

17 hydrophobic with increasing temperature, and such property has been harnessed to obtain well-

18 known pluronic hydrogels. ${ }^{24}$ In our synthesis scheme, hydrophobic PPGDA has been introduced 19 in the gel network to maintain a balance with hydrophilic PAAc and PMAM. As a result, hydrogels 20 with high stretchability and resilience, capable of performing power amplified activities, were 21 obtained.

22 For the present study, we have considered $17 \%, 27 \%$, and $37 \%\left(\mathrm{~g} \mathrm{~mL}^{-1}\right)$ total monomer (MAM 23 and AAc) concentration while keeping a constant MAM to AAc weight ratio of 1:4. A fixed 
1 proportion of KPS $\left(0.2 \%\left(\mathrm{~g} \mathrm{~g}^{-1}\right)\right)$ and PPGDA $\left(1 \%\left(\mathrm{~g} \mathrm{~g}^{-1}\right)\right)$ with respect to the total monomer weight

2 was used for each gel. The FTIR results of the synthesized gel capture the copolymerization of the 3 monomers (see Supporting Information, Figure S1). Based on the monomer concentration, these 4 gels are referred to as 17\%-, 27\%-, and 37\%-Gels throughout this article. The water content of the 5 as-prepared $17 \%-, 27 \%$-, and $37 \%$-Gels has been determined as $80.9 \pm 0.9 \%, 67.6 \pm 0.2 \%$, and $664.9 \pm 0.1 \%$, respectively, by drying the gel samples in a vacuum oven for $72 \mathrm{~h}$ at room temperature 7 (Figure S2A-B and Table S1). During gel formation, we have observed some level of syneresis for 8 the $17 \%$ - and $27 \%$-Gels likely related to the presence of hydrophobic monomers, therefore, the 9 polymer concentrations in those gels have been found to be slightly higher than the monomer 10 concentration in the initial solution.

11 No significant additional swelling was observed when the as-prepared samples were stored in a $120.8 \mathrm{M}$ aqueous $\mathrm{NaCl}$ solution. However, submerging the samples in water results in significant 13 swelling. Specifically, the dried 17\%-, 27\%-, and 37\%-Gel samples immersed in water for $24 \mathrm{~h}$ 14 can absorb water $\approx 87.9 \pm 14.9,44.2 \pm 1.4$, and $34.6 \pm 0.1$ times of their dry weight, respectively 15 (Figure S2C and Table S1). The water absorbance capacity of our gel is higher than that of PAAc16 PMAM hydrogels,${ }^{25}$ however, it is comparable to that of acrylamide-based polyampholyte 17 hydrogels. ${ }^{26}$ High water absorption capacity in our gels can be related to their synthesis conditions. 18 The presence of cations from $\mathrm{NaCl}$ screens the acid groups of $\mathrm{AAc}$ from ionization, ${ }^{25}$ as a result, 19 the swelling of the synthesized gel is relatively lower. However, when the samples are submerged 20 in water, $\mathrm{NaCl}$ salts leach out, resulting in ionization of acid groups and the corresponding increase 21 of water uptake. We have found that the samples are stable in $\mathrm{NaCl}$ solution, even for months, 22 therefore, these gels can be used in a saline environment. In this study, we have considered the as23 prepared samples for further testing. As the gels display a significant swelling capacity when 
1 submerged in water, it can be hypothesized that the polymer chains are loosely crosslinked, with

2 high molecular weight strands between the crosslinks that can be extended significantly during the 3 mechanical stretching as discussed below.

4 The structure of our gels can be compared with resilin, a disorderly protein that consists of both 5 hydrophilic and hydrophobic segments. ${ }^{3}$ At low strain, the resilin elasticity is governed by the

6 entropic stretching of hydrophilic segments (exon I) ${ }^{1-4}$ In contrast, the high-strain response is

7 primarily governed by the stretching of hydrophobic segments (exon III). ${ }^{1-4}$ The hydrophobic

8 content dictates the water content in the gel, therefore, affects the modulus of the material. The

9 power amplification mechanism in many biological systems can be attributed to the rapid

10 retraction response of resilin from a stretched state. Such a response is not only dictated by the

11 entropic spring-like response of hydrophilic chains but also by the behavior of hydrophobic

12 stretched chains in an aqueous environment. In the stretched state, the hydrophobic segments in

13 contact with water, an unfavorable solvent, rapidly return to their original collapsed condition once

14 the strain is removed. However, the enthalpic penalty is not significant, therefore, resilin is highly

15 resilient. For our gels, we expect to see similar mechanical responses of the hydrophilic and

16 hydrophobic segments mimicking the mechanical properties of resilin. 
(A)

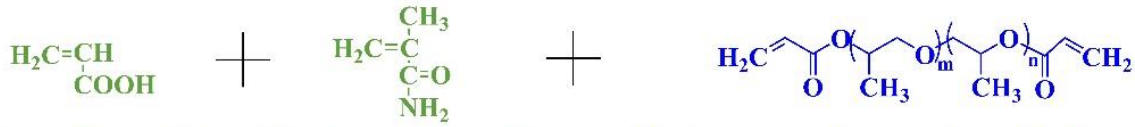

Acrylic acid Methacrylamide Poly(propylene glycol) diacrylate

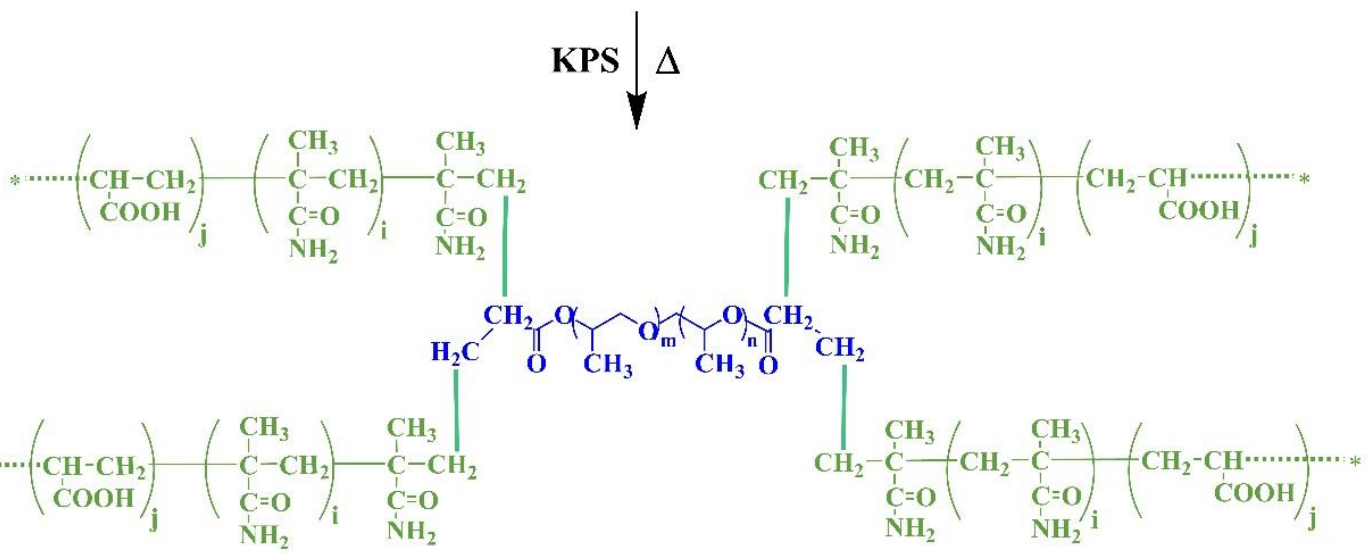

\section{Crosslinked polymer}
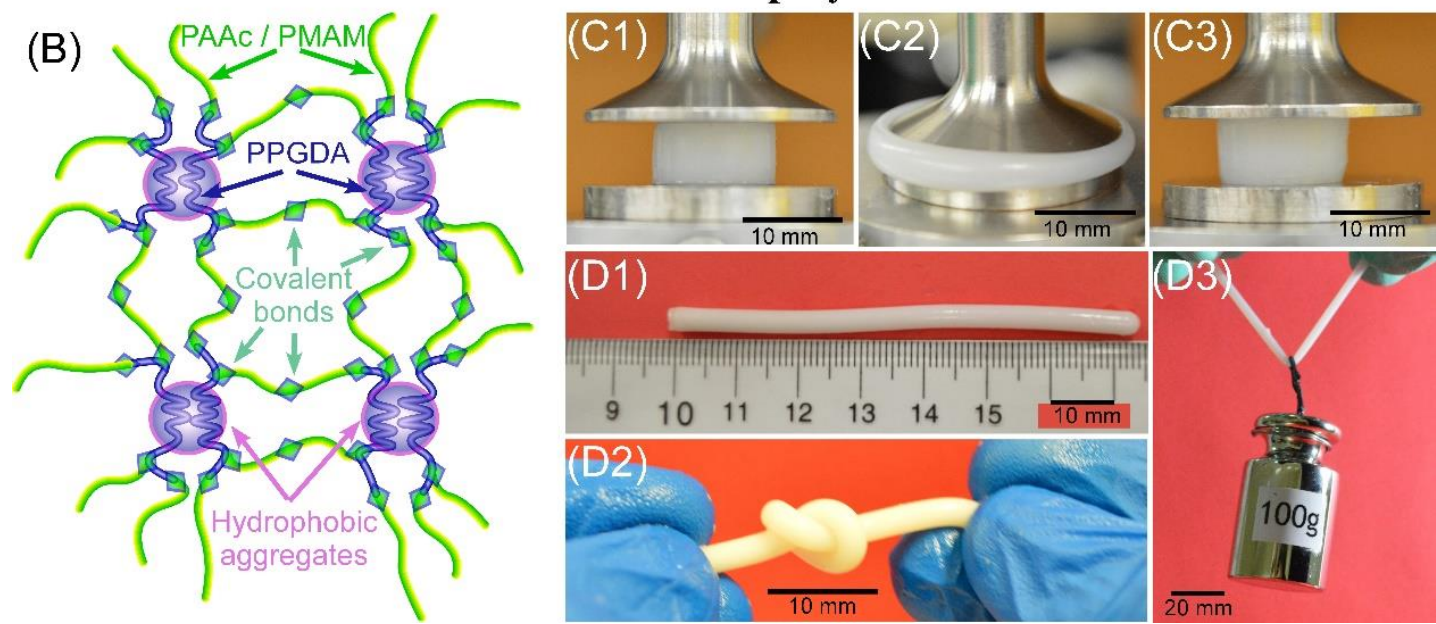

2 Figure 1. Chemical structure and stretchability of hydrogel. (A) Reaction scheme for the hydrogel

3 formation. (B) The three-dimensional structure of hydrogel, where hydrophobic aggregates (pink

4 spheres) containing PPGDA molecules (blue chains) are connected by the hydrophilic PAAc and PMAM chains (green chains). Here, the green diamonds represent covalent bonds. (C)

6 Compression of a $27 \%$-Gel sample representing the undeformed state (C1), after compression

7 (C2), and after removing the stress (C3). (D) Complex deformation of a gel string (37\%-Gel) in

8 an undeformed state (D1), forming a knot (D2), and supporting $100 \mathrm{~g}$ deadweight (D3). 


\section{$1 \quad$ Mechanical properties}

2 The synthesized gels are highly compressible and resilient. As displayed in Figure 1C, a 3 cylindrical sample with an initial height of $5 \mathrm{~mm}$ was compressed to $1 \mathrm{~mm}$ with a strain-rate of

$4 \quad 0.2 \mathrm{~s}^{-1}$, and the sample quickly recovered to the original height with a negligible residual strain

5 after the compressive strain was released. The sample has an ability to return to its original form

6 even after undergoing a complex deformation (Figure 1D1-2). The sample can also bear a

7 concentrated load without failing (Figure 1D3).

8 As the samples display excellent resilience behavior in compression mode, the stretchability and

9 gel modulus have been determined using tensile testing at room temperature $\left(22^{\circ} \mathrm{C}\right)$ utilizing a

10 custom-built instrument developed in our research group. ${ }^{15,16}$ Figure 2A shows nominal stress

$11(\sigma)$ as a function of strain $(\varepsilon)$ for $17 \%$-, 27\%-, and 37\%-Gels obtained from these tensile

12 experiments using a strain-rate $(\dot{\mathcal{E}}) \approx 0.048 \mathrm{~s}^{-1}$. The $\sigma$ was calculated considering the initial cross-

13 sectional area of the sample gauge region (see Figure S3). To determine the strain values

14 accurately, we have used an image analysis technique, where the distance between the lines drawn

15 on the sample as a function of time was used to estimate $\varepsilon$ and $\dot{\varepsilon}$ (Figure 2B and Figure S3). ${ }^{15,16}$

16 This technique allows us to determine strain values more accurately in comparison to the

17 traditional methods, where the displacement of the sample clamp or a stage is tracked to determine

18 the strain values, possibly leading to an erroneous higher stretchability of the gel samples. ${ }^{27}$ Our

19 gel samples display a typical s-shaped response of elastomers ${ }^{12,28}$ and a high stretchability before

20 failing, $\varepsilon_{f}$ (Figure $2 \mathrm{C}$ ). For example, the $\varepsilon_{f}$ for the $17 \%$-Gel is as high as $\approx 8.6$. Images of the

21 stretched gels with three different polymer concentrations are displayed in Figure S4A-C for

22 comparison. In addition, the experiments were also performed at $\dot{\varepsilon} \approx 0.48 \mathrm{~s}^{-1}$, however, no 
1 noticeable change in the $\sigma-\varepsilon$ behavior was observed by increasing $\dot{\varepsilon}$ an order of magnitude

2 (Figure S4D), indicating low energy dissipation in these gels.

3 The $\sigma-\varepsilon$ responses display higher initial slopes with increasing polymer concentration,

4 indicating an increase in modulus. To determine the small-strain tensile modulus $(E)$, the neo-

5 Hookean model expressed as $\sigma=(E / 3)\left((\varepsilon+1)-(\varepsilon+1)^{-2}\right)$ for the uniaxial loading was fitted over

6 the range of $0 \leq \varepsilon \leq 0.35$ (Figure S5). ${ }^{28}$ The estimated $E$ values are 15.1, 56.8, and $103.1 \mathrm{kPa}$ for the

7 17\%-, 27\%-, and 37\%-Gel, respectively (Figure 2D). These values are comparable to the other

8 crosslinked hydrogels like polyacrylamide, ${ }^{29}$ hydrophobically modified acrylic gels, ${ }^{22}$

9 alginate/polyacrylamide, ${ }^{30}$ or gelatin hydrogels. ${ }^{31}$ With the increase in monomer concentration, a

10 higher modulus ( $d \sigma / d \lambda$ ) can be noticed, however, the $\varepsilon_{f}$ decreases with increasing monomer

11 concentration. The $\varepsilon_{f}$ values of $27 \%$-Gel and $37 \%$-Gel are not significantly different, evaluated

12 using a t-test with $95 \%$ confidence interval. Note that these two gels display similar swelling

13 behavior. Also, the failure stress $\left(\sigma_{f}\right)$ values of $17 \%$ - and $27 \%$-Gels are not statistically different.

14 Since we have maintained a constant monomer to crosslinker ratio, an increase in monomer

15 concentration increases the crosslinking density leading to a higher gel modulus. ${ }^{28}$ Strain-

16 stiffening behavior observed in all three gels at high $\varepsilon$ can be related to the finite-extensibility of

17 the chains ${ }^{32,33}$ as most likely, the hydrophobic aggregates dissociate, and the PPG chains are

18 stretched. Strain-stiffening allows these gels to carry a concentrated load, as seen in Figure 1D3,

19 despite not having a very high $E$.

20 In determining the resilience behavior of these gels, cyclic loading tests were performed on three

21 gel samples considered here. Although it is preferred to stretch the samples to a prescribed strain,

22 real-time strain measurements from the images have been found to be difficult. Therefore, the 
1 samples were stretched to a prescribed stress value using $\dot{\varepsilon} \approx 0.048 \mathrm{~s}^{-1}$ and then unloaded to zero

2 stress with the same $\dot{\varepsilon}$. After completion of one loading cycle, the stress was continuously

3 increased by $10 \mathrm{kPa}$ for the next cycle until the sample failed, mostly at the $\varepsilon_{f}$ values obtained

4 from the tensile tests (Figure 2C). Results for the 37\%-Gel for particular stress values are shown

5 in Figure 2E-F. The loading and unloading cycles for all gels with $10 \mathrm{kPa}$ step-increase are shown

6 in Figure S6, S7, and S8. The strain-recovery after unloading is almost complete, as the residual

7 strain has been found to be negligible, especially at the high-stretch values and for higher monomer

8 concentration. For example, the residual strain for all gel samples is $\approx 5.7 \%$ for the $\sigma=30 \mathrm{kPa}$

9 cycle, however, it reduces to $2 \%$ for $37 \%$-Gel for the $\sigma=130 \mathrm{kPa}$ cycle. The actual strain-recovery

10 can be a little higher, particularly for the low applied stress, as we have a few percentages of

11 measurement error in capturing the position of marked lines $(\approx 0.2 \mathrm{~mm}$ error in measuring distances

12 between the lines on the samples). The resilience behavior of these gels can be quantified as the

13 ratio of energy recovered during the unloading cycle to the energy absorbed during the loading

14 cycle. A remarkable resilience of $\approx 98 \%$ is captured for $37 \%$-Gel (Table S2). With a decrease in

15 the monomer concentration, the resilience reduces to $90.3 \%$ for $27 \%$-Gel and $88.6 \%$ for $17 \%$-Gel.

16 Overall, we have obtained less than $10 \%$ energy loss during a cycle, which is not very significant.

17 The high resilience of our gels can mostly be attributed to the reversible stretching of hydrophilic

18 chains. As discussed above, because of the presence of $\mathrm{NaCl}$, the acid groups are mostly screened,

19 enhancing the stretchability of the chains. ${ }^{21}$ When subjected to a load, the stretching of hydrophilic

20 chains between two crosslinks occurs, followed by the dissociation of hydrophobic aggregates and

21 subsequent stretching of hydrophobic blocks, as the entropic penalty in chain stretching is lower

22 than overcoming the hydrophobic association energy. ${ }^{15,16}$ Once the load is removed, in addition to

23 the hydrophilic chains, the hydrophobic chains also retract quickly from the unfavorable aqueous 
1 environment to form aggregates again, leading to high resilience and almost full strain recovery.

2 The low hysteresis loop also indicates that the enthalpic loss associated with dissociation and

3 reformation of hydrophobic aggregates is not significant, similar to that observed in the resilin.

4 Therefore, the low molecular weight of the hydrophobic PPG-chains is beneficial. ${ }^{10,34}$ In addition

5 to the enthalpic contribution because of association and dissociation of hydrophobic blocks, the

6 hysteresis in our gels can also be attributed to the viscous dissipation caused by the inhomogeneity

7 and defects in the microstructure.

8 The resilience values of the present gels are comparable to those obtained for recombinant resilin

9 and elastin with the resilience of $\approx 97 \%$ and $90 \%$, respectively. ${ }^{1}$ The performance of our gels can

10 also be compared with other synthetic hydrogels displaying high stretchability with low hysteresis.

11 A majority of these systems is either polyethylene glycol (PEG) based hydrogels ${ }^{9,35,36}$ or polymer-

12 clay nanocomposite hydrogels. ${ }^{10,34}$ The tetra-PEG systems display high stretchability and low

13 hysteresis, however, their synthesis requires a relatively complex reaction route. ${ }^{9,35}$ In comparison,

14 PEG-based systems incorporated with hydrophobic polydimethylsiloxane blocks display relatively

15 high modulus. ${ }^{9}$ These systems display very high resilience $(\approx 98 \%)$, but the stretchability can be

16 limited. ${ }^{9}$ Polymer-clay nanocomposite hydrogels display high stretchability and low hysteresis

17 over a limited range of strain, however, these gels can have a longer recovery time from the highly

18 stretched state. ${ }^{10,34}$ In contrast, our gels recover instantaneously, even at high strain. The

19 instantaneous recovery of our samples was further explored through the retraction experiments. 
(A)

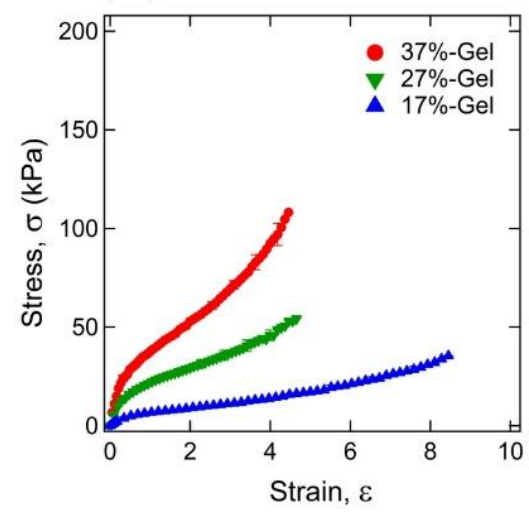

(D)

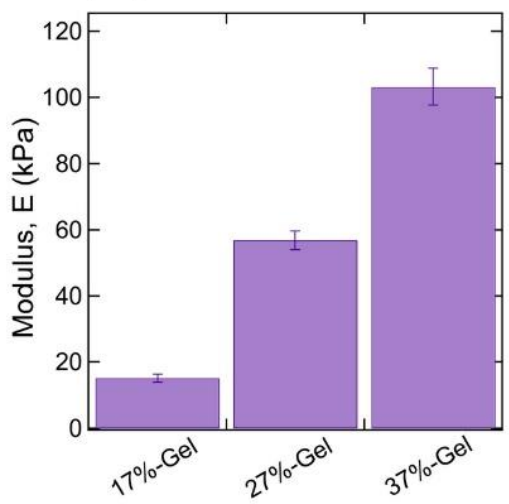

(B2)

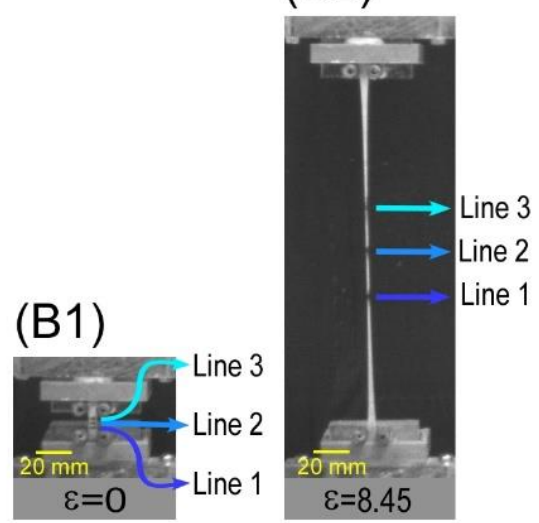

(E)

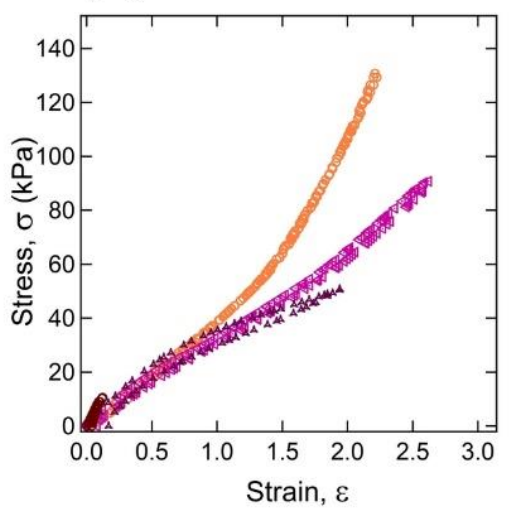

(C)

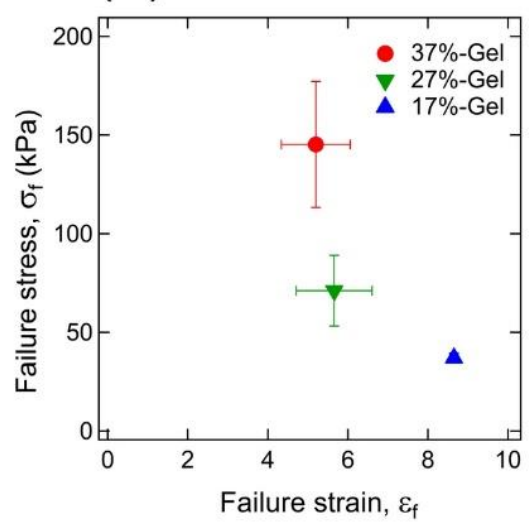

$(\mathrm{F})$

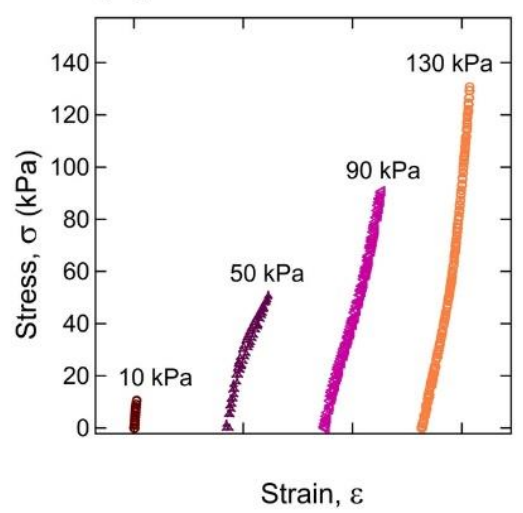

2 Figure 2. Tensile testing and cyclic loading experiments. (A) Nominal stress $(\sigma)$ as a function of

3 strain $(\varepsilon)$ for 17\%-, 27\%-, and 37\%-Gels. Results are an average of three runs with standard

4 deviation. (B) Images of the 17\%-Gel sample during a tensile experiment: (B1) initial condition

$5(\varepsilon=0)$, and (B2) before the failure of the gel, capturing high stretchability of the sample ( $\varepsilon=8.45)$.

6 (C) Failure stress $\left(\sigma_{f}\right)$ and failure strain $\left(\varepsilon_{f}\right)$ for the three gels. The error bars represent the

7 standard deviation. (D) Young's modulus of the three gels obtained by fitting the neo-Hookean

8 model to the experimental data up to $\varepsilon \approx 0.35$. The error bars represent the standard deviation. (E-

9 F) Results from cyclic loading experiments for 37\%-Gel plotted for the maximum stress values,

$10 \sigma=10,30,90$, and $130 \mathrm{kPa}$. Each cycle is shifted by $0,4,8$, and 12 units, respectively, along the

11 strain-axis for clarity. 


\section{Retractability of hydrogels.}

2 Figure 3 shows the results of retraction experiments performed on a 37\%-Gel string using the

3 stretch ratio $(\lambda=\varepsilon+1)$ of 6 . Here, the stretched string was cut at the bottom clamp using a pair of

4 scissors, and then the string was allowed to retract freely. The sample was marked with seven

5 equidistant lines (Figure 3A1-A2) to track the position of lines with time as the sample retracted.

6 The line positions were tracked from the release of the string until the time it slacked. The results

7 presented here correspond to Line 1, which achieved the highest velocity and acceleration during

8 the retraction. The position of Line 1 for all three gels are plotted as a function of time in Figure

$93 \mathrm{~B}$ for $\lambda=6$. The response of Line 1 can be divided into three regions. First, the initial plateau for

10 a few milliseconds (ms) indicating that the string is still in the static condition. Next, as time

11 progresses, the sample starts to retract. It accelerates from the static condition to the peak velocity

12 resulting in curvature in the position vs. time data. ${ }^{8,37-41}$ A sharper curvature signifies a higher

13 acceleration value. Finally, the string reaches a constant velocity displayed by a straight line with

14 a constant slope.

15 The line position with time was fitted with a fifth-order Fourier series (Figure S9), and the

16 obtained function was then differentiated for estimating velocity and acceleration (see Supporting

17 Information). The estimated velocity values for the three gels are shown in Figure 3C, capturing

18 static condition, acceleration, and constant velocity. The estimated acceleration values are shown

19 in Figure 3D representing the peak values corresponding to the curvature in the position-time plot.

20 Note that other functions, such as cubic spline, can be used to fit the experimental data, but we

21 found that Fourier series with sufficient number of coefficients ( $5^{\text {th }}$ order considered here) and

22 with an appropriate value of $\omega$, the data can be fitted reasonably well. Figure $3 \mathrm{E}$ and $\mathrm{F}$ summarize

23 the maximum velocity and acceleration for the $\lambda$ values of $3-6$. With the increase in monomer 
1 concentration, both velocity and acceleration increase suggesting that higher values can be

2 obtained by further increasing the monomer concentration or partially drying the samples. The 3 maximum velocity and acceleration for $37 \%$-Gel and $\lambda=6$ are $\approx 16 \mathrm{~m} \mathrm{~s}^{-1}$ and $\approx 4 \times 10^{3} \mathrm{~m} \mathrm{~s}^{-2}$, 4 respectively.

5 The retraction velocity values are comparable to those of a frog jump $\left(\approx 4.5 \mathrm{~m} \mathrm{~s}^{-1}\right)$, froghopper 6 jump $\left(\approx 4.7 \mathrm{~m} \mathrm{~s}^{-1}\right)$, and Mantis shrimp appendage strike $\left(\approx 30 \mathrm{~m} \mathrm{~s}^{-1}\right){ }^{5}$ The acceleration is similar to

7 the jump of a froghopper $\left(\approx 5.4 \times 10^{3} \mathrm{~m} \mathrm{~s}^{-2}\right) .{ }^{5}$ The retraction velocity in our gel is lower than dry, 8 vulcanized natural rubber $\left(\approx 125 \mathrm{~m} \mathrm{~s}^{-1}\right)^{37}$ but somewhat similar to the polyurethane elastomers $(\approx 25$ $\left.9 \mathrm{~m} \mathrm{~s}^{-1}\right) .^{5}$ To the best of our knowledge, this is the first demonstration of achieving such a high 10 velocity and acceleration in hydrogels.

11 The retraction mechanism in elastomers has been previously studied in detail using a very similar 12 experimental protocol described above. ${ }^{8,37-40}$ A sudden release of the lower end of a sample from 13 a stretched state causes the propagation of a retraction pulse or stress wave with a velocity $v$ in the 14 longitudinal direction. ${ }^{37,39,40}$ Note that the Line 1 velocity $(u)$ is different and is lower than $v$, as $u$ 15 originates from the momentum generated by the retraction pulse. It can be shown that $16 u=\sqrt{\frac{\sigma(\lambda-1)}{\rho}}$ and $v=\lambda \sqrt{\frac{\sigma}{\rho(\lambda-1)}}$ from momentum balance. ${ }^{37}$ Here, $\lambda$ is the stretch ratio from 17 which the sample is released, and $\sigma$ is the corresponding stress value, and $\rho$ is the density of the 18 system. Note that for linear elastic material with a modulus $E, u=(\lambda-1) \sqrt{E / \rho}$, and $v=\lambda \sqrt{E / \rho}$ 19 , as reported in the literature. ${ }^{38,39}$ These relationships corresponding to linear elastic materials are 20 not suitable for our case as the modulus of our system is changing with applied stretch (Figure $212 \mathrm{~A}$ ). Note that in isotropic media, the sound velocity can be estimated as $\sqrt{K / \rho}$, independent of $22 \lambda$, where $K$ is the bulk modulus of the material. ${ }^{40}$ In the retraction experiments, the retraction 
1 pulse is dictated by the $E$ values, therefore, $u$ and $v$ values are typically a couple of orders of

2 magnitude lower than the sound velocity in elastomers. ${ }^{39}$

3 The free retraction velocity of our sample was then compared with the theoretically estimated $u$

4 value, $u=\sqrt{\sigma(\lambda-1) / \rho} \cdot{ }^{39,41,37}$ We have used density, $\rho \approx 1.05,1.10$, and $1.14 \mathrm{~g} \mathrm{~mL}^{-1}$ for $17 \%$-,

$527 \%$-, and $37 \%$-Gel, respectively. $\sigma$ corresponding to a particular $\lambda$ was estimated from Figure

62 A. The density of each gel was determined by measuring the mass of the as-prepared cubic-

7 shaped sample and the volume occupied by that sample. Figure $3 \mathrm{E}$ compares $u$ as a function of $\lambda$

8 with the experimentally obtained values of Line 1 for each gel.

9 Here, the predicted $u$ values are 1.1-1.7 times higher than the experimentally obtained retraction

10 velocity of Line 1 but are of the same order of magnitude. The equation used for estimating $u$

11 originates from the momentum balance, therefore, it does not account for any dissipation in the

12 system. The lower retraction velocity in our system than that predicted theoretically can be related

13 to the viscous dissipation that can arise because of the presence of defects in the gel microstructure.

14 For the higher polymer concentration, an increase in the number of defects can be anticipated,

15 resulting in a higher deviation from the theoretical prediction. The cyclic loading experiments

16 show a very small amount of hysteresis or dissipation, interestingly, that small dissipation may

17 have caused the reduction in retraction velocity from the theoretical prediction for a perfectly

18 elastic material. This dissipation is also likely to lower the acceleration values in our gels compared

19 to that observed in some biological systems $\approx 10^{5} \mathrm{~m} \mathrm{~s}^{-2 .}{ }^{5}$ 
(A1)

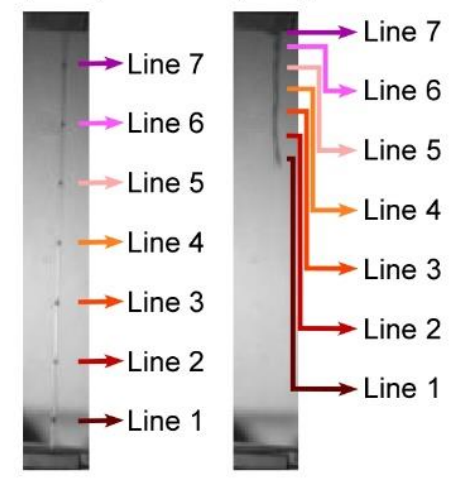

(D)

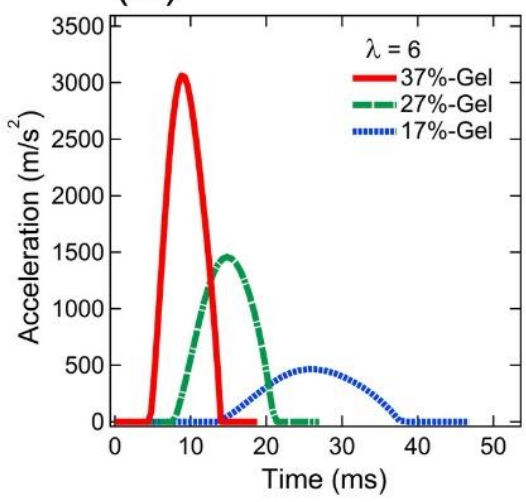

(B)

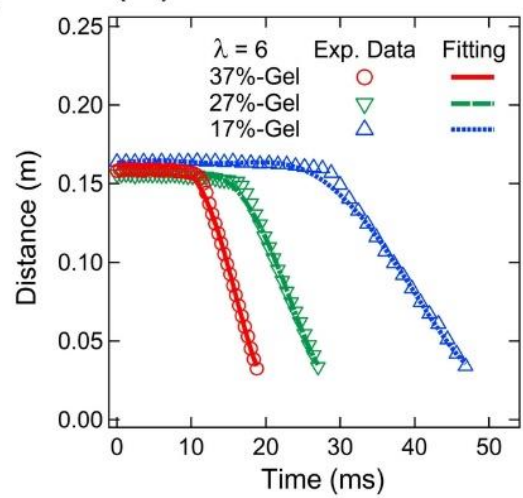

(E)

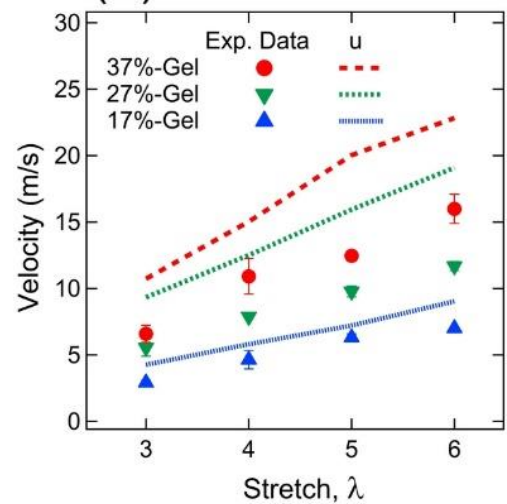

(C)

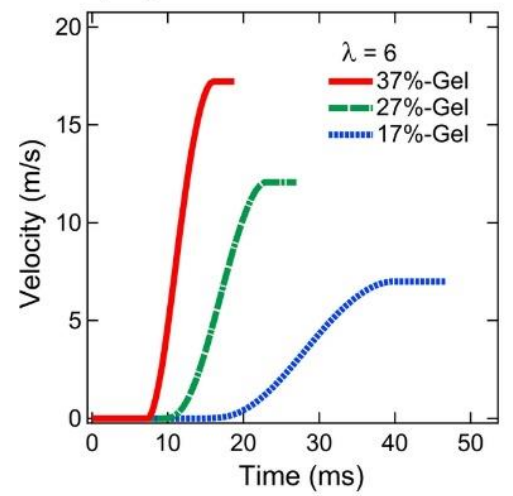

$(\mathrm{F})$

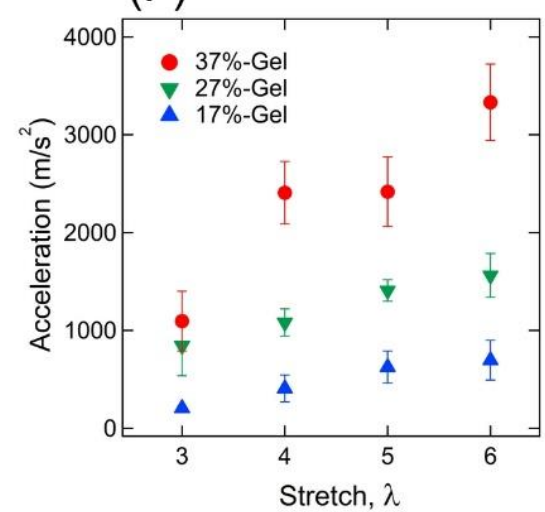

2 Figure 3. Retraction of gel samples from a stretched-state. Image of a 37\%-Gel string: (A1) at

3 the stretched condition, $\lambda=6$ at $t=0$ ms., marked with 7 lines (Line 1-7), and (A2) fully retracted

4 after $t \approx 19$ ms. (B) Position, (C) velocity, and (D) acceleration of Line 1 as a function of time for

5 all gels with $\lambda=6$. In (B) both the experimental data and the fitting of the Fourier series are shown.

6 (E) Retraction velocity as a function of different $\lambda$ for all three gels. A comparison of the

7 experimentally observed velocity of Line 1 with theoretically predicted retraction velocity $(u)$ as a

8 function of $\lambda$. (F) Maximum acceleration corresponding to Line 1 as a function of $\lambda$ for all three

9 gels. The error bars represent the standard deviation.

10 
Solid projectile launching by hydrogels. Since our gels have high stretchability and resilience,

2 we have shown their applications for catapulting solid projectiles. As shown in Figure 4, by using

3 a $37 \%$-Gel string, we have been able to launch a solid projectile of $1.53 \mathrm{~g}$ to a distance $(L)$ of

$47.96 \pm 0.24 \mathrm{~m}$. The achieved projectile velocity was $15.93 \pm 0.63 \mathrm{~m} \mathrm{~s}^{-1}$, and $93 \%$ of the energy stored

5 in the system during stretching was converted into kinetic energy. This was achieved for $\lambda \approx 6$,

6 and different $L$ values can be obtained by considering the different $\lambda$ values. Also, the sample

7 can be reused multiple times. This further opens up novel applications of the gels reported here.
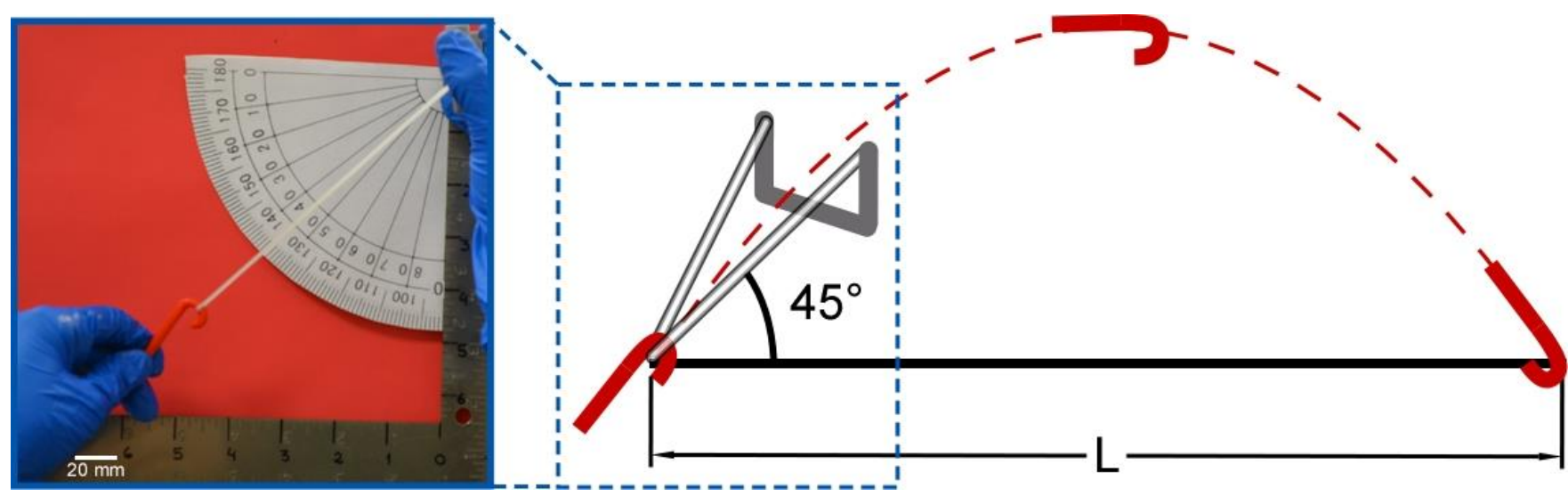

10 Figure 4. Application of gels for launching a projectile. Image and scheme of a projectile

11 launching by using a 37\%-Gel for stretch, $\lambda=6$.

\section{Conclusions}

14 In conclusion, we report a novel hydrogel synthesized through a simple free radical 15 copolymerization scheme. By selecting an appropriate ratio of hydrophilic PAAc and PMAM 16 chains and hydrophobic associations of PPGDA chains, highly stretchable $(\lambda \approx 8.6)$ and resilient 17 gels $(\approx 98 \%)$ with a negligible residual strain $(\approx 2 \%)$ have been obtained. After released from a 
1 stretched state, the gel samples achieve retraction velocity, as high as $\approx 16 \mathrm{~m} \mathrm{~s}^{-1}$ and acceleration

$2 \approx 4 \times 10^{3} \mathrm{~m} \mathrm{~s}^{-2}$. These properties resemble naturally occurring elastomeric biopolymers, like resilin.

3 High resilience and stretchability can be utilized in many activities, such as catapulting a projectile

4 or other power amplification systems. Because of a simple synthetic route, the hydrogel properties

5 can be further tuned, and new applications can be envisioned. Further, based on the results

6 presented here, we can hypothesize that the design of materials aimed for power amplified

7 activities requires (1) high modulus to density ratio, (2) high stretchability, and (3) minimal energy

8 dissipation or high energy recovery.

10 ASSOCIATED CONTENT

11 Supporting Information: Fourier transform infrared spectroscopy of monomers and gel;

12 Images of swelling behavior of gels; Scheme and images of the dimensions of dogbone samples

13 with marked-lines; Images and data of gel stretchability and strain-rate dependence; Graph

14 corresponding to the determination of the tensile modulus of gels; cyclic loading curves of 17\%-,

$1527 \%$-, and 37\%-Gels; Graph corresponding to the marked-line positions as a function of time

16 during retraction and fitted parameters corresponding to Line 1 for $37 \%$-Gel at stretch of 6 . Tables

17 of the results of swelling behavior and resilience of gels.

\section{AUTHOR INFORMATION}

20 Corresponding Author

21 *Santanu Kundu. E-mail: santanukundu@che.msstate.edu 


\section{Author contributions}

2 S.K. proposed and supervised this project. S.K., R.M.B.P., and S.M. analyzed the data and wrote

3 the paper. R.M.B.P., S.M., and B.M. performed the experiments. R.W. and S.M.H. conducted

4 preliminary experiments. All authors have given approval to the final version of the manuscript.

\section{ACKNOWLEDGMENT}

7 This research is supported by the National Science Foundation (DMR-1352572, DMR8 2004501).

\section{REFERENCES}

10 (1) Elvin, C. M.; Carr, A. G.; Huson, M. G.; Maxwell, J. M.; Pearson, R. D.; Vuocolo, T.;

11 Liyou, N. E.; Wong, D. C. C.; Merritt, D. J.; Dixon, N. E. Synthesis and Properties of 12 Crosslinked Recombinant Pro-Resilin. Nature 2005, 437 (7061), 999-1002. 13 https://doi.org/10.1038/nature04085.

14 (2) Nairn, K. M.; Lyons, R. E.; Mulder, R. J.; Mudie, S. T.; Cookson, D. J.; Lesieur, E.; Kim, 15 M.; Lau, D.; Scholes, F. H.; Elvin, C. M. A Synthetic Resilin Is Largely Unstructured. 16 Biophys. J. 2008, 95 (7), 3358-3365. https://doi.org/10.1529/biophysj.107.119107.

17 (3) Qin, G.; Hu, X.; Cebe, P.; Kaplan, D. L. Mechanism of Resilin Elasticity. Nat. Commun.

(4) Su, R. S.-C.; Kim, Y.; Liu, J. C. Resilin: Protein-Based Elastomeric Biomaterials. Acta Biomater. 2014, 10 (4), 1601-1611. https://doi.org/10.1016/j.actbio.2013.06.038. 
1 (5) Ilton, M.; Bhamla, M. S.; Ma, X.; Cox, S. M.; Fitchett, L. L.; Kim, Y.; Koh, J.;

Krishnamurthy, D.; Kuo, C.-Y.; Temel, F. Z.; Crosby, A. J.; Prakash, M.; Sutton, G. P.; Wood, R. J.; Azizi, E.; Bergbreiter, S.; Patek, S. N. The Principles of Cascading Power Limits in Small, Fast Biological and Engineered Systems. Science (80-. ). 2018, 360 (6387), eaao1082. https://doi.org/10.1126/science.aao1082.

(6) Patek, S. N.; Korff, W. L.; Caldwell, R. L. Deadly Strike Mechanism of a Mantis Shrimp. Nature 2004, 428 (6985), 819-820. https://doi.org/10.1038/428819a.

(7) Gosline, J.; Lillie, M.; Carrington, E.; Guerette, P.; Ortlepp, C.; Savage, K. Elastic Proteins: Biological Roles and Mechanical Properties. Philos. Trans. R. Soc. London. Ser. B Biol. Sci. 2002, 357 (1418), 121-132. https://doi.org/10.1098/rstb.2001.1022.

(8) Ilton, M.; Cox, S. M.; Egelmeers, T.; Sutton, G. P.; Patek, S. N.; Crosby, A. J. The Effect of Size-Scale on the Kinematics of Elastic Energy Release. Soft Matter 2019, 15 (46), 95799586. https://doi.org/10.1039/C9SM00870E.

(9) Cui, J.; Lackey, M. A.; Madkour, A. E.; Saffer, E. M.; Griffin, D. M.; Bhatia, S. R.; Crosby, A. J.; Tew, G. N. Synthetically Simple, Highly Resilient Hydrogels. Biomacromolecules 2012, 13 (3), 584-588. https://doi.org/10.1021/bm300015s.

(10) Su, X.; Mahalingam, S.; Edirisinghe, M.; Chen, B. Highly Stretchable and Highly Resilient Polymer-Clay Nanocomposite Hydrogels with Low Hysteresis. ACS Appl. Mater. Interfaces 2017, 9 (27), 22223-22234. https://doi.org/10.1021/acsami.7b05261.

(11) Tjin, M. S.; Low, P.; Fong, E. Recombinant Elastomeric Protein Biopolymers: Progress and Prospects. Polym. J. 2014, 46 (8), 444-451. https://doi.org/10.1038/pj.2014.65. 
1 (12) Sun, T. L.; Kurokawa, T.; Kuroda, S.; Ihsan, A. Bin; Akasaki, T.; Sato, K.; Haque, M. A.;

Nakajima, T.; Gong, J. P. Physical Hydrogels Composed of Polyampholytes Demonstrate High Toughness and Viscoelasticity. Nat. Mater. 2013, 12 (10), 932-937. https://doi.org/10.1038/nmat3713.

(13) Gao, G.; Du, G.; Sun, Y.; Fu, J. Self-Healable, Tough, and Ultrastretchable Nanocomposite Hydrogels Based on Reversible Polyacrylamide/Montmorillonite Adsorption. ACS Appl. Mater. Interfaces 2015, 7 (8), 5029-5037. https://doi.org/10.1021/acsami.5b00704.

(14) Xiang, S.; Li, T.; Dong, W.; Lu, Q. A Facile Method to Fabricate Tough Hydrogel with Ultra-Wide Adjustable Stiffness, Stress, and Fast Recoverability. J. Polym. Sci. Part B Polym. Phys. 2018, 56 (21), 1469-1474. https://doi.org/10.1002/polb.24737.

(15) Mishra, S.; Badani Prado, R. M.; Lacy, T. E.; Kundu, S. Investigation of Failure Behavior of a Thermoplastic Elastomer Gel. Soft Matter 2018, 14 (39), 7958-7969. https://doi.org/10.1039/C8SM01397G.

(16) Mishra, S.; Badani Prado, R. M.; Zhang, S.; Lacy, T. E.; Gu, X.; Kundu, S. Mechanical Properties and Failure Behavior of Physically Assembled Triblock Copolymer Gels with Varying Midblock Length. J. Polym. Sci. Part B Polym. Phys. 2019, 57 (15), 1014-1026. https://doi.org/10.1002/polb.24860.

(17) Sammalkorpi, M.; Karttunen, M.; Haataja, M. Ionic Surfactant Aggregates in Saline Solutions: Sodium Dodecyl Sulfate (SDS) in the Presence of Excess Sodium Chloride ( $\mathrm{NaCl})$ or Calcium Chloride ( $\mathrm{CaCl} 2$ ). J. Phys. Chem. B 2009, 113 (17), 5863-5870. https://doi.org/10.1021/jp901228v. 
1 (18) Hooper, H. H.; Baker, J. P.; Blanch, H. W.; Prausnitz, J. M. Swelling Equilibria for

Positively Ionized Polyacrylamide Hydrogels. Macromolecules 1990, 23 (4), 1096-1104. https://doi.org/10.1021/ma00206a031.

(19) Yang, M.; Liu, C.; Li, Z.; Gao, G.; Liu, F. Temperature-Responsive Properties of Poly(Acrylic Acid-Co-Acrylamide) Hydrophobic Association Hydrogels with High Mechanical Strength. Macromolecules 2010, 43 (24), 10645-10651. https://doi.org/10.1021/ma1022555.

(20) Zhang, F.; Guo, Z.; Gao, H.; Li, Y.; Ren, L.; Shi, L.; Wang, L. Synthesis and Properties of Sepiolite/Poly (Acrylic Acid-Co-Acrylamide) Nanocomposites. Polym. Bull. 2005, 55 (6), 419-428. https://doi.org/10.1007/s00289-005-0458-2.

(21) Walker, A.; Vratsanos, M.; Kozawa, S.; Askew, T.; Hemmendinger, K.; McGrail, B.; Bedford, N.; Wnek, G. Enhanced Elasticity in Poly(Acrylic Acid) Gels via Synthesis in the Presence of High Concentrations of Select Salts. Soft Matter 2019, 15 (38), 7596-7604. https://doi.org/10.1039/C9SM01101C.

(22) Miquelard-Garnier, G.; Hourdet, D.; Creton, C. Large Strain Behaviour of Nanostructured Polyelectrolyte Hydrogels. Polymer (Guildf). 2009, $50 \quad$ (2), 481-490. https://doi.org/10.1016/j.polymer.2008.11.045.

(23) Liu, X.; Duan, L.; Gao, G. Rapidly Self-Recoverable and Fatigue-Resistant Hydrogels Toughened by Chemical Crosslinking and Hydrophobic Association. Eur. Polym. J. 2017, 89 (October 2016), 185-194. https://doi.org/10.1016/j.eurpolymj.2017.02.025.

(24) Alexandridis, P.; Alan Hatton, T. Poly(Ethylene Oxide)-Poly(Propylene Oxide)- 
Poly(Ethylene Oxide) Block Copolymer Surfactants in Aqueous Solutions and at Interfaces: Thermodynamics, Structure, Dynamics, and Modeling. Colloids Surfaces A Physicochem. Eng. Asp. 1995, 96 (1-2), 1-46. https://doi.org/10.1016/0927-7757(94)03028-X.

(25) Dubey, S.; Bajpai, S. K. Poly(Methacrylamide-Co-Acrylic Acid) Hydrogels for Gastrointestinal Delivery of Theophylline. I. Swelling Characterization. J. Appl. Polym. Sci. 2006, 101 (5), 2995-3008. https://doi.org/10.1002/app.23542.

(26) Baker, J. P.; Stephens, D. R.; Blanch, H. W.; Prausnitz, J. M. Swelling Equilibria for Acrylamide-Based Polyampholyte Hydrogels. Macromolecules 1992, 25 (7), 1955-1958. https://doi.org/10.1021/ma00033a019.

(27) Mrozek, R. A.; Leighliter, B.; Gold, C. S.; Beringer, I. R.; Yu, J. H.; VanLandingham, M. R.; Moy, P.; Foster, M. H.; Lenhart, J. L. The Relationship between Mechanical Properties and Ballistic Penetration Depth in a Viscoelastic Gel. J. Mech. Behav. Biomed. Mater. 2015, 44, 109-120. https://doi.org/10.1016/j.jmbbm.2015.01.001.

(28) Treloar, L. R. G. The Physics of Rubber Elasticity; Oxford University Press, USA, 1975.

(29) Kundu, S.; Crosby, A. J. Cavitation and Fracture Behavior of Polyacrylamide Hydrogels. Soft Matter 2009, 5 (20), 3963. https://doi.org/10.1039/b909237d.

(30) Sun, J.-Y.; Zhao, X.; Illeperuma, W. R. K.; Chaudhuri, O.; Oh, K. H.; Mooney, D. J.; Vlassak, J. J.; Suo, Z. Highly Stretchable and Tough Hydrogels. Nature 2012, 489 (7414), 133-136. https://doi.org/10.1038/nature11409.

(31) Frieberg, B. R.; Garatsa, R.-S.; Jones, R. L.; Bachert, J. O.; Crawshaw, B.; Liu, X. M.; Chan, E. P. Viscoplastic Fracture Transition of a Biopolymer Gel. Soft Matter 2018, 14 (23), 
4696-4701. https://doi.org/10.1039/C8SM00722E.

2

(32) Hashemnejad, S. M.; Kundu, S. Nonlinear Elasticity and Cavitation of a Triblock Copolymer Gel. Soft Matter 2015, $11 \quad$ (21), 4315-4325. https://doi.org/10.1039/C5SM00330J.

(33) Erk, K. A.; Henderson, K. J.; Shull, K. R. Strain Stiffening in Synthetic and Biopolymer Networks. Biomacromolecules 2010, $11 \quad$ (5), $1358-1363$. https://doi.org/10.1021/bm100136y.

(34) Zhu, M.; Liu, Y.; Sun, B.; Zhang, W.; Liu, X.; Yu, H.; Zhang, Y.; Kuckling, D.; Adler, H.J. P. A Novel Highly Resilient Nanocomposite Hydrogel with Low Hysteresis and Ultrahigh Elongation. Macromol. Rapid Commun. 2006, 27 (13), 1023-1028. https://doi.org/10.1002/marc.200600159.

(35) Kamata, H.; Akagi, Y.; Kayasuga-Kariya, Y.; Chung, U. -i.; Sakai, T. "Nonswellable" Hydrogel Without Mechanical Hysteresis. Science (80-. ). 2014, 343 (6173), 873-875. https://doi.org/10.1126/science.1247811.

(36) Sakai, T.; Akagi, Y.; Matsunaga, T.; Kurakazu, M.; Chung, U.; Shibayama, M. Highly Elastic and Deformable Hydrogel Formed from Tetra-Arm Polymers. Macromol. Rapid Commun. 2010, 31 (22), 1954-1959. https://doi.org/10.1002/marc.201000286.

(37) Tunnicliffe, L. B.; Thomas, A. G.; Busfield, J. J. C. The Free Retraction of Natural Rubber: A Momentum-Based Model. Polym. Test. 2015, 47, 36-41. https://doi.org/10.1016/j.polymertesting.2015.07.012.

(38) James, H. M.; Guth, E. Theory of the Retraction of Stressed Rubber. Phys. Rev. 1944, 66 
2

14

16

17

18

19

15

(39) Gent, A. N.; Marteny, P. The Effect of Strain upon the Velocity of Sound and the Velocity of Free Retraction for Natural Rubber. J. Appl. Phys. 1982, 53 (9), 6069-6075. https://doi.org/10.1063/1.331558.

(40) Bogoslovov, R. B.; Roland, C. M. Viscoelastic Effects on the Free Retraction of Rubber. J. Appl. Phys. 2007, 102 (6), 063531. https://doi.org/10.1063/1.2784018.

(41) Mason, P.; A, P. R. S. L. Finite Elastic Wave Propagation in Rubber. Proc. R. Soc. London. Ser. A. Math. Phys. Sci. 1963, 272 (1350), 315-330. https://doi.org/10.1098/rspa.1963.0056.

0

1

12

3

4


Table of Contents (TOC) graphic
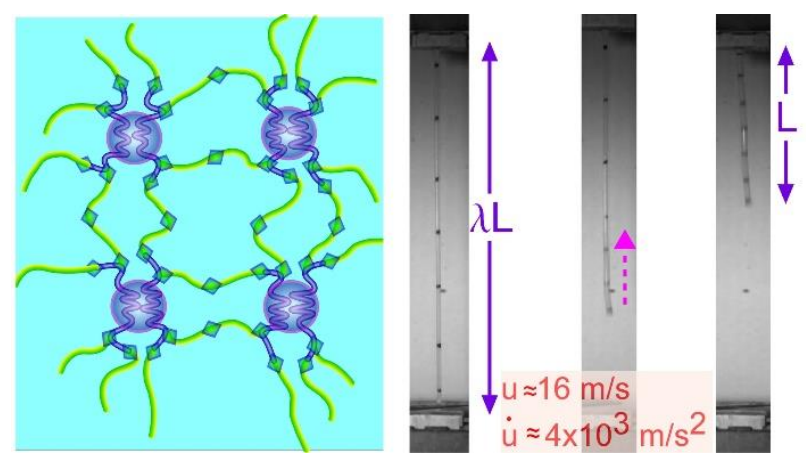


\title{
Supporting Information
}

\section{Achieving high-speed retraction in a stretchable hydrogel}

\author{
Rosa Maria Badani Prado, Satish Mishra, Buckston Morgan, Rangana Wijayapala, \\ Seyed Meysam Hashemnejad, Santanu Kundu*
}

Dave C. Swalm School of Chemical Engineering, 323 Presidents Circle, Mississippi State University, MS State, MS 39762, USA.

\author{
Corresponding Author \\ *Santanu Kundu. E-mail: santanukundu@che.msstate.edu
}




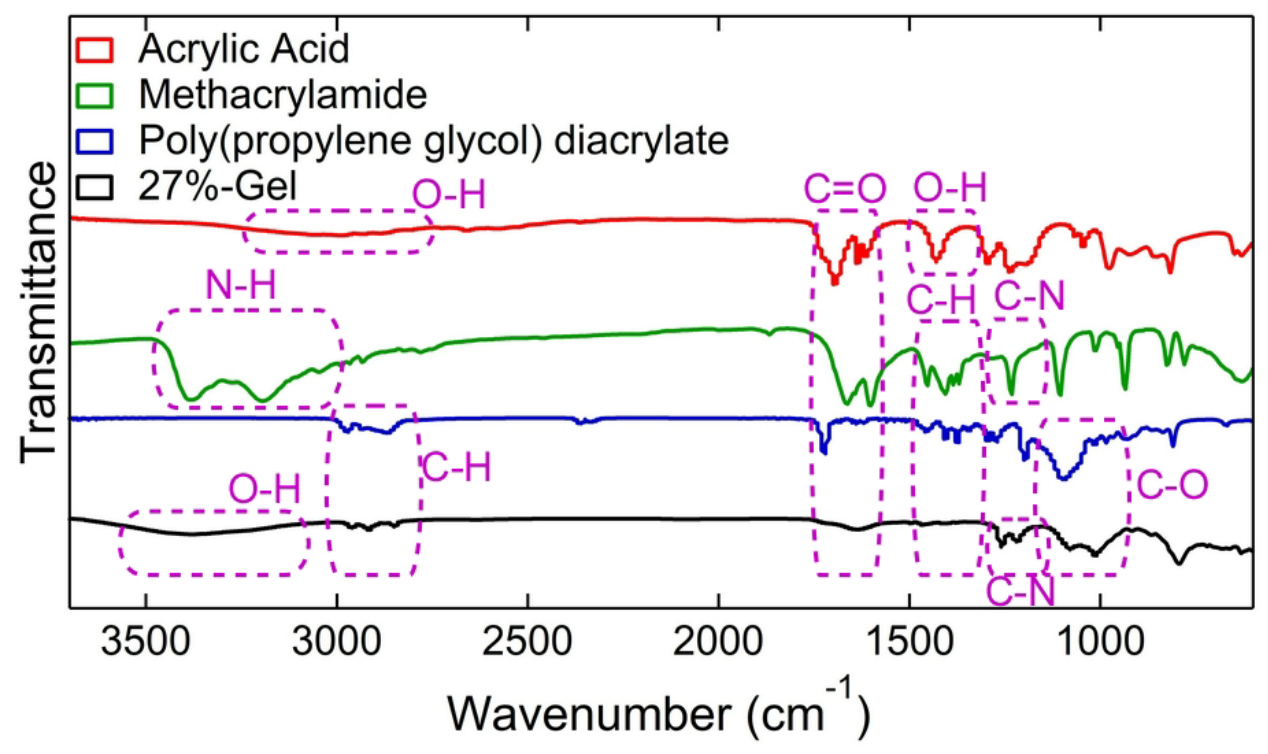

Figure S1. FTIR spectrum of acrylic acid, methacrylamide, poly(propylene glycol) diacrylate, and 27\%-Gel.

The spectrum of acrylic acid (AAc) displays a broad band at $\approx 2988 \mathrm{~cm}^{-1}$ attributed to the O-H stretching, a peak at $\approx 1431 \mathrm{~cm}^{-1}$ corresponding to $\mathrm{O}-\mathrm{H}$ bending, and a peak at $\approx 1695 \mathrm{~cm}^{-1}$ for $\mathrm{C}=\mathrm{O}$ stretching, all related to the carboxyl groups. ${ }^{1}$ Methacrylamide (MAM) spectrum displays peaks for the amide group at $\approx 2195 \mathrm{~cm}^{-1}$ and $3385 \mathrm{~cm}^{-1}$ attributed to the $\mathrm{N}-\mathrm{H}$ stretching along with a peak at $\approx 1660 \mathrm{~cm}^{-1}$ for the $\mathrm{C}=\mathrm{O}$ stretching and at $\approx 1230 \mathrm{~cm}^{-1}$ for the $\mathrm{C}-\mathrm{N}$ stretching. ${ }^{2}$ It also has peaks at $\approx 1400$ and $1450 \mathrm{~cm}^{-1}$ attributed to the $\mathrm{C}-\mathrm{H}$ bending. Poly(propylene glycol) diacrylate (PPGDA) spectrum displays peaks at $\approx 2869$ and $2971 \mathrm{~cm}^{-1}$ attributed to the C-H stretching, at $\approx 1374$ and $1405 \mathrm{~cm}^{-1}$ attributed to the $\mathrm{C}-\mathrm{H}$ bending, at $\approx 1722 \mathrm{~cm}^{-1}$ for $\mathrm{C}=\mathrm{O}$ stretching, and at $\approx 1096 \mathrm{~cm}^{-1}$ for the C-O stretching. ${ }^{3}$ The crosslinking and polymerization of the compounds can be understood from the spectrum ${ }^{1,2}$ of $27 \%-G e l$, where (1) a broad band is shown at $\approx 3375 \mathrm{~cm}^{-1}$ attributed to O-H stretching likely overlapped with N-H stretching, both related to the AAc, MAM, and PPGDA interaction, (2) peaks at $\approx 2849$ and $2961 \mathrm{~cm}^{-1}$ attributed to the $\mathrm{C}$-H stretching related 
to the presence of PPGDA in the structure, (3) a peak of low intensity at $\approx 1635 \mathrm{~cm}^{-1}$ related to $\mathrm{C}=\mathrm{O}$ stretching coming from the presence of all the compounds, (4) a peak with low intensity at $\approx 1467 \mathrm{~cm}^{-1}$ attributed to the C-H bending likely related to the crosslinking of the compounds, (5) peaks at $\approx 1219 \mathrm{~cm}^{-1}$ and $1258 \mathrm{~cm}^{-1}$ for C-N stretching possibly related to the crosslinked MAM, (6) and a peak at $\approx 1080 \mathrm{~cm}^{-1}$ for the $\mathrm{C}-\mathrm{O}$ stretching related to the presence of PPGDA. 


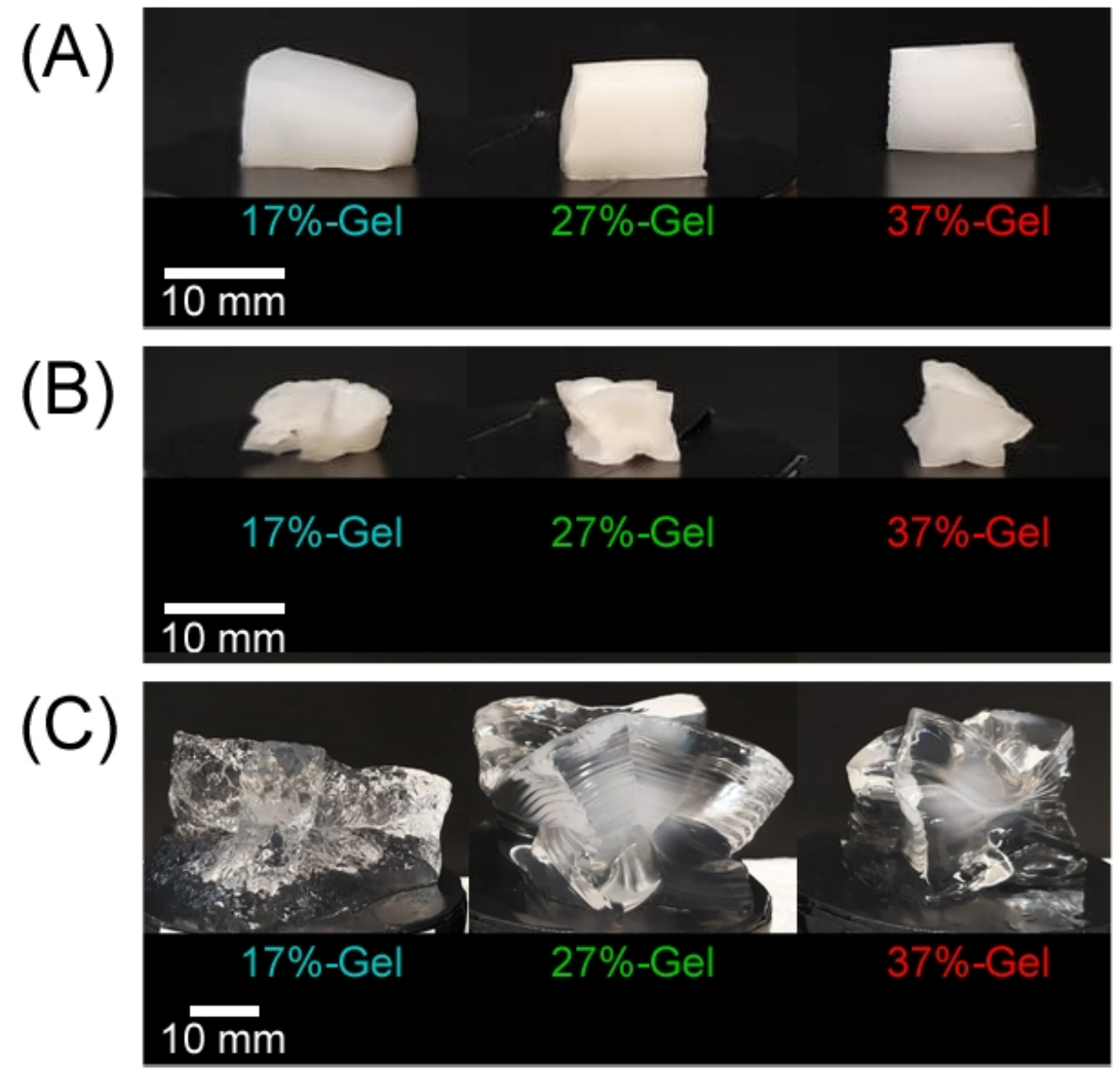

Figure S2. Swelling behavior of gels. (A) As-prepared 17\%-, 27\%-, and 37\%-Gels. (B) Dried gels after 72 h of drying. (C) Gels after swelling for $24 \mathrm{~h}$. 


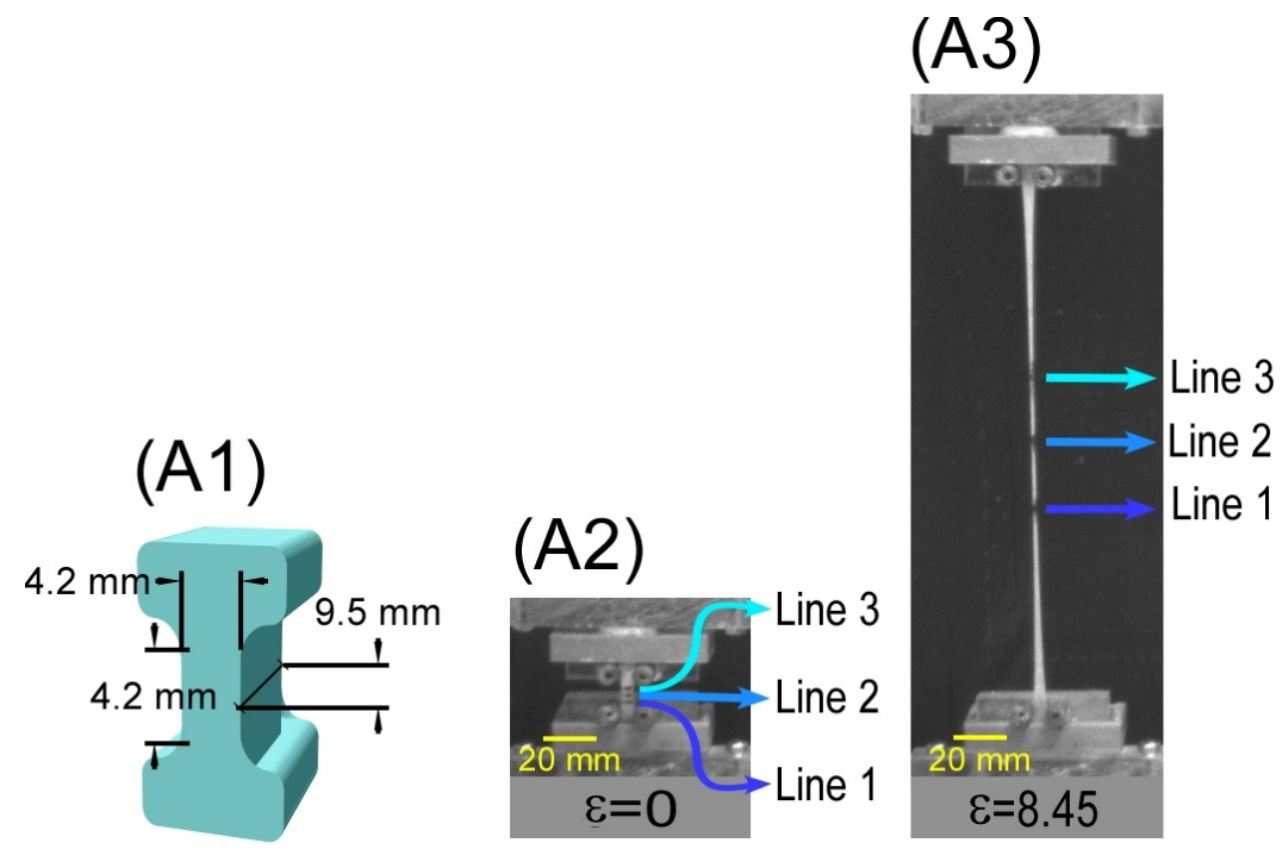

Figure S3. Dimensions of dogbone samples with marked-lines. (A1) Schematic of the dogboneshape specimen for tensile experiments representing the dimensions. Images of tensile testing indicating the marked lines on the sample $(17 \%-\mathrm{Gel})$ that were used to measure the strain $(\varepsilon)$ and strain-rate ( $\dot{\varepsilon}$ ). (A2) Unstretched sample, $\varepsilon=0$, and (A3) stretched sample, $\varepsilon=8.45$. 

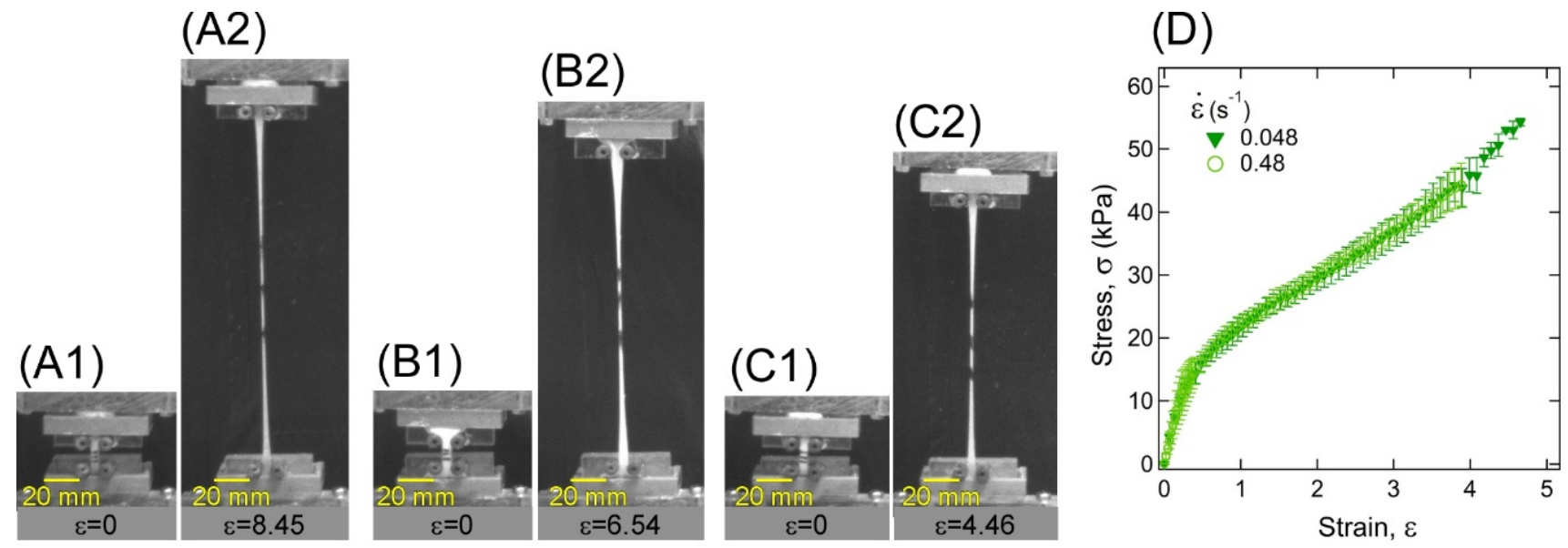

Figure S4. Gel stretchability and strain-rate dependence. Images of a 17\%-Gel at the unstretched condition ( $\varepsilon=0$ ) (A1) and the maximum stretched condition ( $\varepsilon=8.45$ ) (A2). Images of 27\%-Gel at the unstretched condition ( $\varepsilon=0)(\mathrm{B} 1)$, and the maximum stretched condition ( $\varepsilon=6.54)(\mathrm{B} 2)$. Images of 37\%-Gel at the unstretched condition ( $\varepsilon=0$ ) (C1), and the maximum stretched condition ( $\varepsilon=4.46$ ) (C2). (D) Nominal stress ( $\sigma$ ) as a function of strain ( $\varepsilon$ ) for a $27 \%$-Gel obtained from tensile testing experiments at strain-rate $(\dot{\varepsilon}) 0.048 \mathrm{~s}^{-1}$ (dark green triangles) and $0.48 \mathrm{~s}^{-1}$ (light green open circles). The error bars represent the standard deviation. 


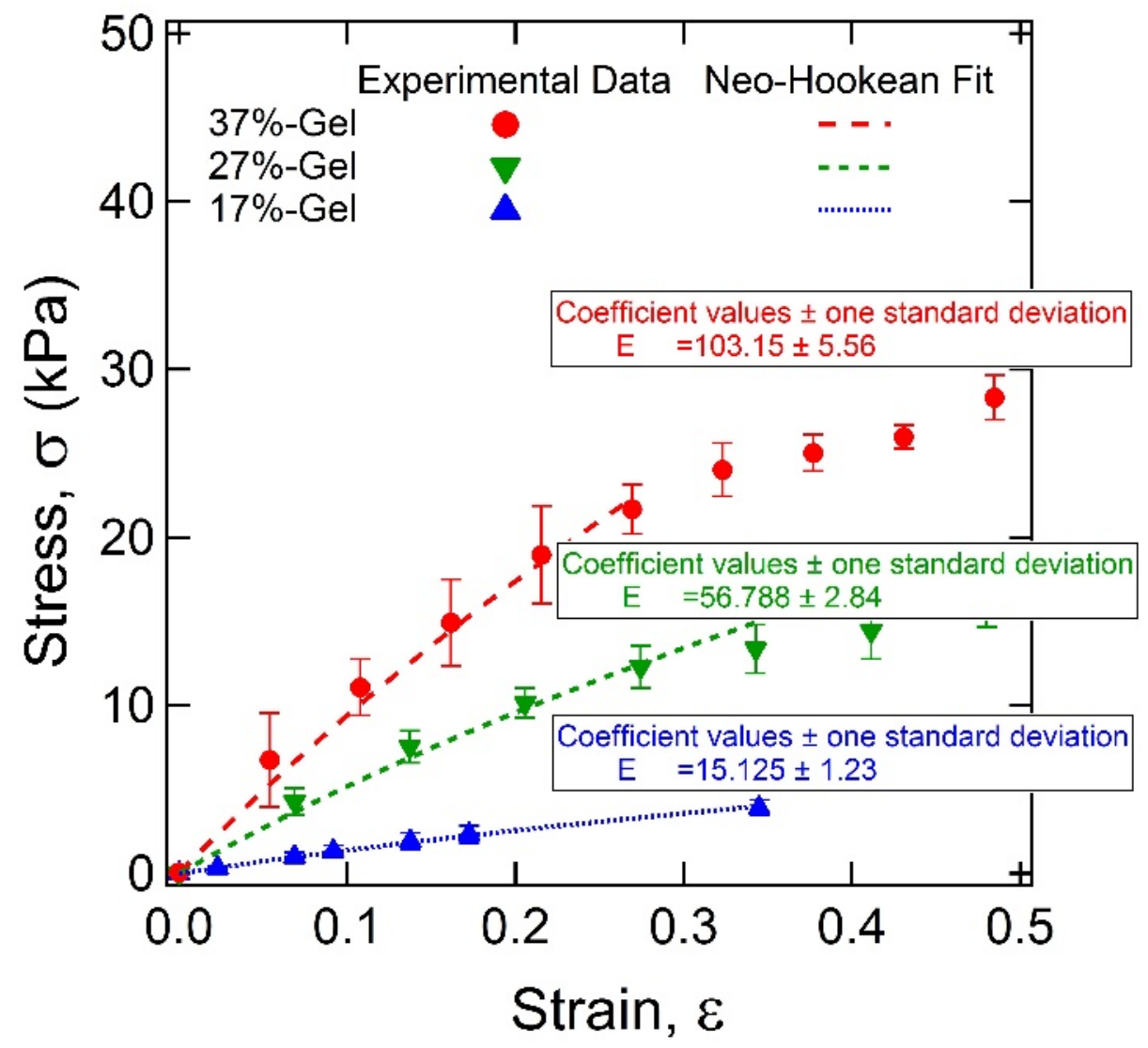

Figure S5. Tensile modulus of gels. Nominal stress $(\sigma)$ as a function of strain $(0 \leq \varepsilon \leq 0.35)$ for 17\%-, 27\%-, and 37\%-Gels fitted with the neo-Hookean model for uniaxial loading. The corresponding tensile modulus (E) values for 17\%-, 27\%-, and 37\%-Gels have been estimated as $\approx 15.1,56.8$, and $103.1 \mathrm{kPa}$, respectively. The error bars represent the standard deviation. 

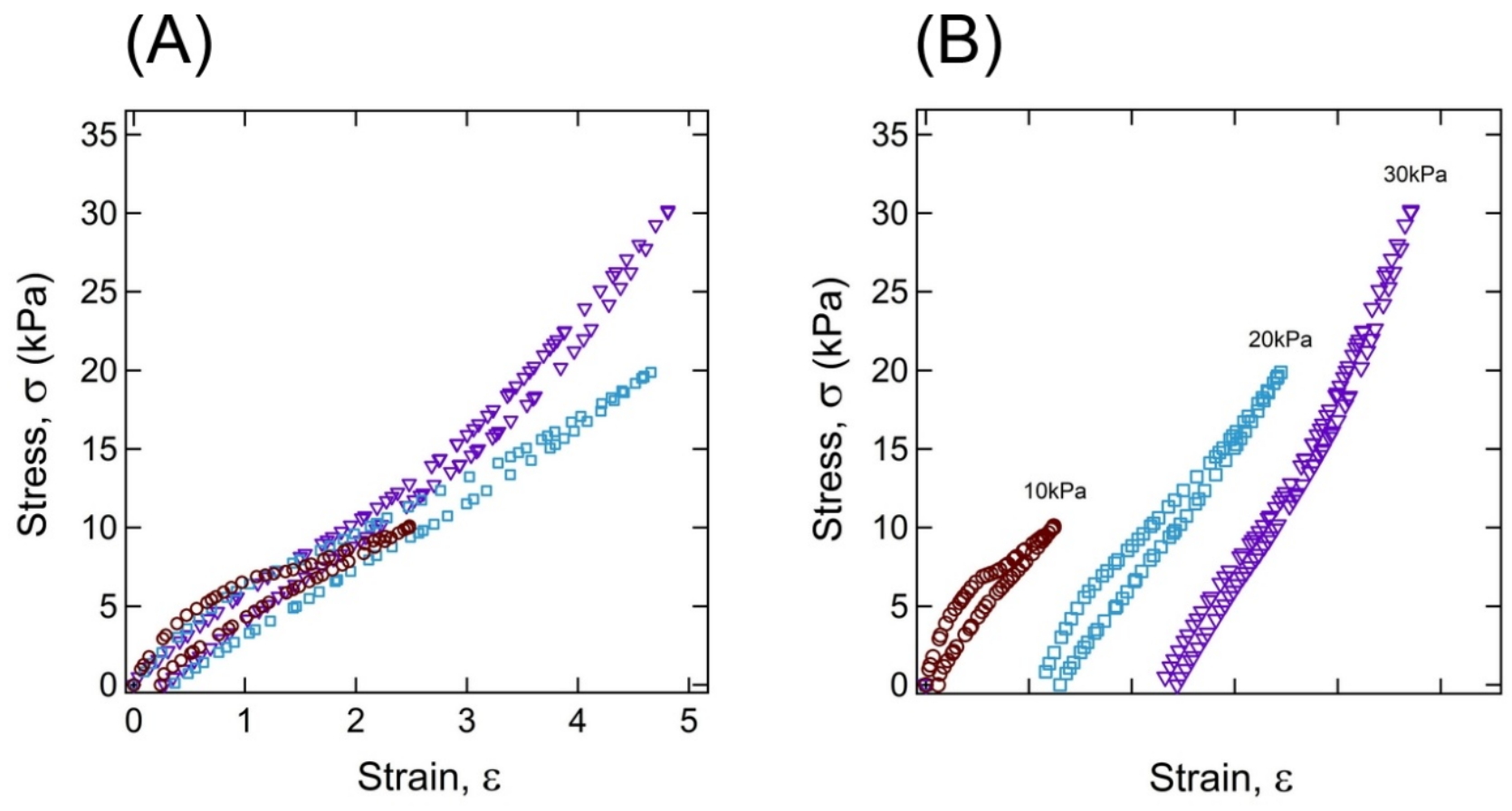

Figure S6. Cyclic loading results of a 17\%-Gel. (A) Nominal stress $(\sigma)$ as a function of strain ( $\varepsilon$ ) obtained from cyclic loading of 10,20 , and $30 \mathrm{kPa}$. (B) Cycles are moved along the $\varepsilon$-axis for clarity and visualization. 

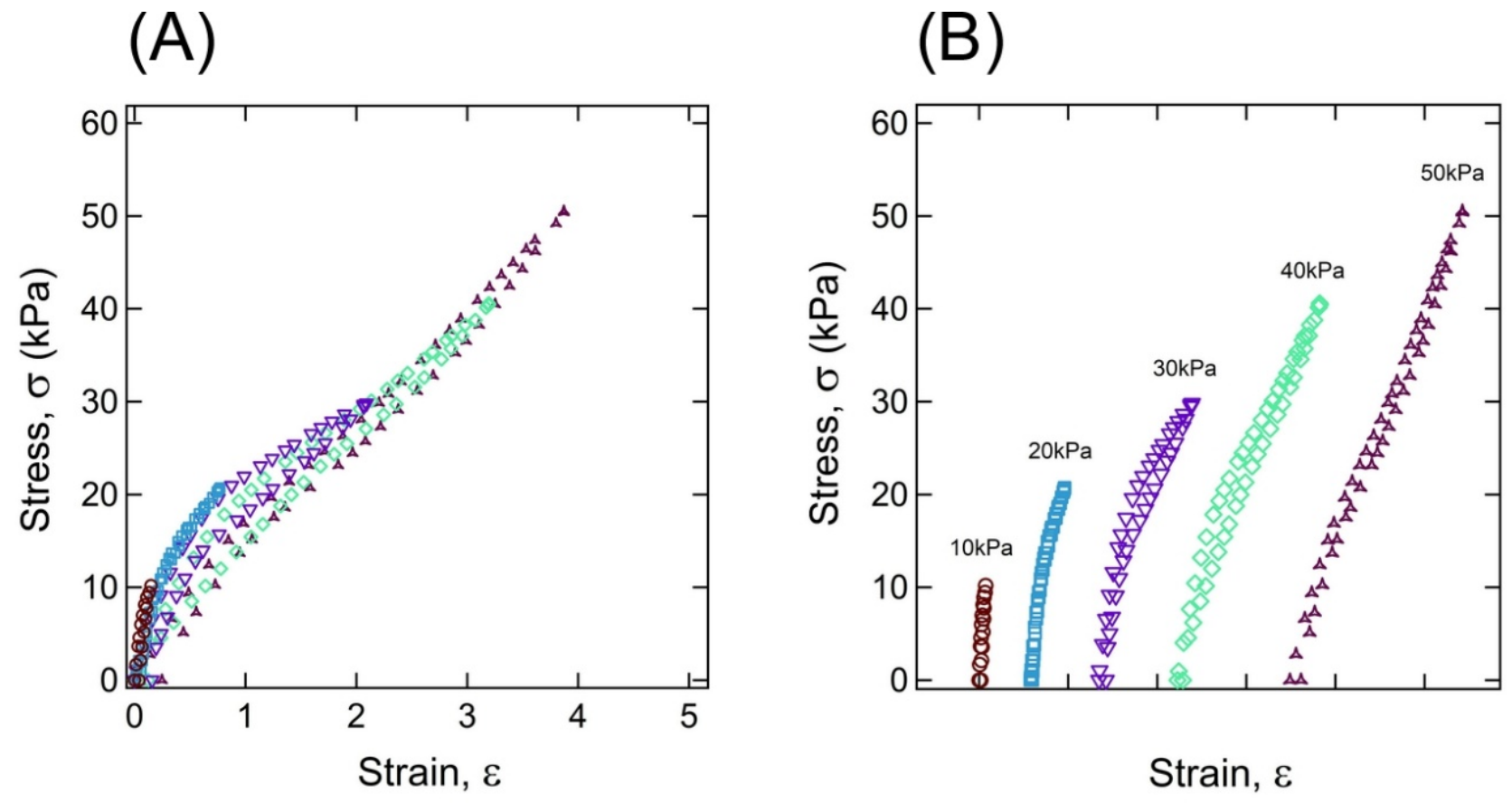

Figure S7. Cyclic loading results of a 27\%-Gel. (A) Nominal stress $(\sigma)$ as a function of strain ( $\varepsilon$ ) obtained from cyclic loading of 10, 20, 30, 40, and $50 \mathrm{kPa}$. (B) Cycles are moved along the $\varepsilon$-axis for clarity and visualization. 
(A)

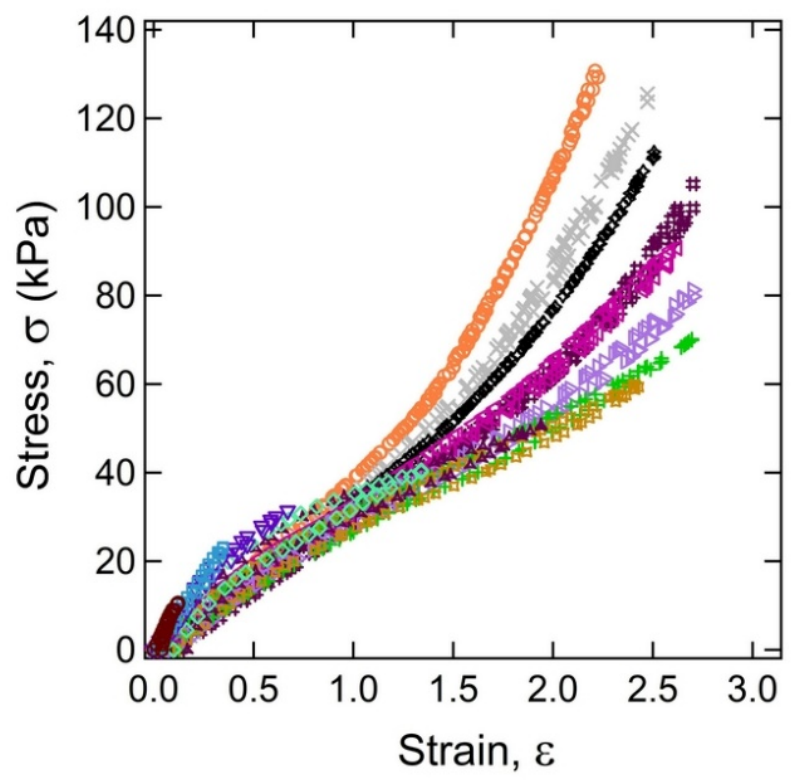

(B)

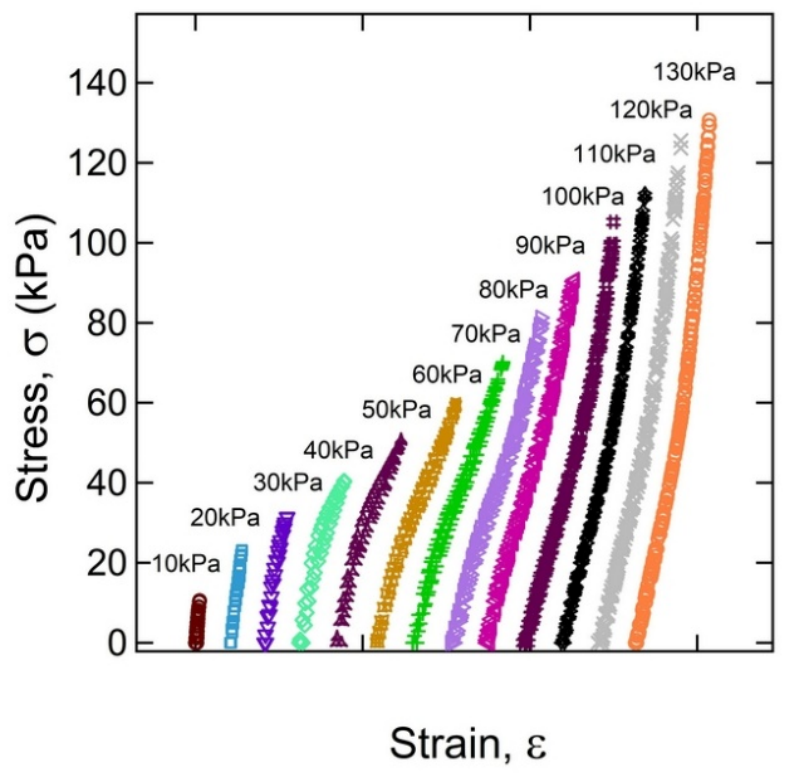

Figure S8. Cyclic loading results of a 37\%-Gel. (A) Nominal stress $(\sigma)$ as a function of strain ( $\varepsilon$ ) obtained from cyclic loading of 10-130 kPa with a step of $10 \mathrm{kPa}$. (B) Cycles are moved along the $\varepsilon$-axis for clarity and visualization. 


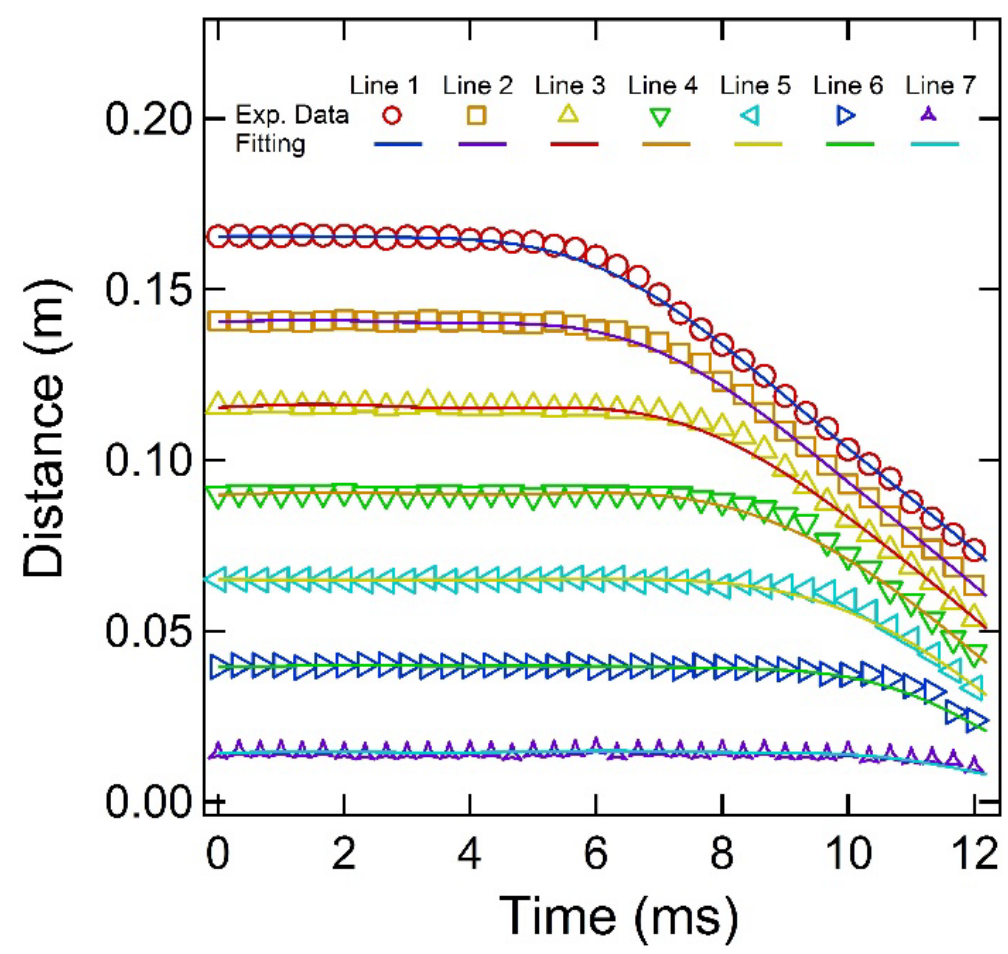

Figure S9. Line positions as a function of time during retraction. The positions of seven marked lines on the samples as a function of time from retraction experiments with $\lambda=6$ for a $37 \%$-Gel sample. The symbols display the values obtained from image processing, and the lines indicate the fifth-order Fourier fit.

The position (f) vs. time (t) data of all lines was fitted with the fifth-order Fourier series expressed as, $f(t)=a_{0}+\sum_{i=1}^{5} a_{i} \cos (\omega t)+\sum_{i=1}^{5} b_{i} \sin (\omega t)$. The obtained function was then differentiated to obtain velocity and acceleration. For example, the fitted parameters obtained for Line 1 for the 37\%-Gel (Figure S9) at $\lambda=6$ are $a_{0}=-1.521 \mathrm{e}+08, \quad a_{1}=2.359 \mathrm{e}+08, \quad a_{2}=-1.056 \mathrm{e}+08, \quad a_{3}=2.237 \mathrm{e}+07$, $a_{4}=-1.064 \mathrm{e}+05, a_{5}=-5.319 \mathrm{e}+05, b_{1}=9.706 \mathrm{e}+07, b_{2}=-1.046 \mathrm{e}+08, b_{3}=5.302 \mathrm{e}+07, b_{4}=-1.338 \mathrm{e}+07$, $b_{5}=1.315 \mathrm{e}+06$, and $\omega=40.32$. The value of $\omega$ provides a time scale of $156 \mathrm{~ms}$. The total time of the retraction experiment was $18 \mathrm{~ms}$, much lower than the experimental time scale, therefore, this $\omega$ value is appropriate for fitting the data. 
Table S1. Results of swelling behavior of gels. The results indicate the water content in asprepared gels and the swelling water capacity.

\begin{tabular}{ccc}
\hline Sample & $\begin{array}{c}\text { Water content in as- } \\
\text { prepared samples } \\
{\left[\%, \mathrm{~g} \mathrm{~g}^{-1}\right]}\end{array}$ & $\begin{array}{c}\text { Swelling ratio after } \\
\text { swell in water } \\
{\left[\mathrm{g} \mathrm{g}^{-1}\right]}\end{array}$ \\
\hline $17 \%-G e l$ & $80.9 \pm 0.9$ & $87.9 \pm 14.9$ \\
$27 \%-G e l$ & $67.6 \pm 0.2$ & $44.2 \pm 1.4$ \\
$37 \%-G e l$ & $64.9 \pm 0.1$ & $34.6 \pm 0.1$ \\
\hline
\end{tabular}

Table S2. Resilience of gels. Maximum stress, strain, and resilience (\%) values for 17\%-, 27\%-, and 37\%-Gels corresponding to the highest stress cycle applied during cyclic-loading experiments.

\begin{tabular}{cccc}
\hline Sample & $\begin{array}{c}\text { Maximum stress } \\
{[\mathrm{kPa}]}\end{array}$ & Maximum strain & $\begin{array}{c}\text { Resilience } \\
{[\%]}\end{array}$ \\
\hline 17\%-Gel & 30 & 4.8 & 88.6 \\
$27 \%-G e l$ & 50 & 3.9 & 90.3 \\
$37 \%-G e l$ & 130 & 2.2 & 97.7 \\
\hline
\end{tabular}

\section{References}

(1) Dubey, S.; Bajpai, S. K. Poly(Methacrylamide-Co-Acrylic Acid) Hydrogels for Gastrointestinal Delivery of Theophylline. I. Swelling Characterization. J. Appl. Polym. Sci. 2006, 101 (5), 2995-3008. https://doi.org/10.1002/app.23542.

(2) Zhang, F.; Guo, Z.; Gao, H.; Li, Y.; Ren, L.; Shi, L.; Wang, L. Synthesis and Properties of Sepiolite/Poly (Acrylic Acid-Co-Acrylamide) Nanocomposites. Polym. Bull. 2005, 55 (6), 419-428. https://doi.org/10.1007/s00289-005-0458-2.

(3) Millipore Sigma. IR Spectrum Table and Chart https://www.sigmaaldrich.com/technicaldocuments/articles/biology/ir-spectrum-table.html (accessed Jun 28, 2020). 